Méthodes nouvelles

\title{
pour le calcul des cheminées d'équilibre
}

\section{New methods for surge-tank calculations}

\author{
PAF B. TTCMAN
}

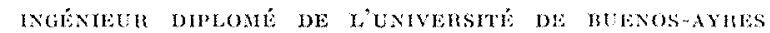

ATTACH AV LABORATORE DAUPHNOIS D'HYDMAULIQUE (NEYRPC)

\begin{abstract}
Equation différentielle. - Avant-projet rapide. - Méthodes graphiques. - Stabilité. - Amplitude limite. - Similitude. - Cheminées d'équilibre avec diaphragme. - Etudes sur modèles réduits. - Autres considérations.
\end{abstract}

\begin{abstract}
Differential equation. - Preliminary project ontline. - Graphical methods. -- Stability. -.. Limit amplitude. - Similitude. — Throttled surge-tanks. - Scale model investigations. other considerations.
\end{abstract}

\section{AVANT-PROPOS}

Le calcul des oscillations en masse dans les systèmes d'amence en charge comportant des chambres d'équilibre se ramène à la résolution d'un système d'équations différentielles relativement simple.

A l'expérience, cette méthode s'est avérée bien satisfaisante, mais malheureusement peu nombreux sont les cas où l'integration directe des equations est possible, ce qui oblige à faire un calcul numérique ou graphique pas à pas. Différents abaques et méthodes de calcul simplifiées permettent cependant d'établir assez rapidement les avant-projets.

Le Laboratoire Dauphinois d'Hydraulique s'est occupé depuis longtemps du calcul de chambres d'équilibre. Les problèmes qui lui ont été posés au cours des vingt dernières années ont permis de mettre au point quelques procédés de ealeul qui réduisent d'une facon parfois considerable le travail materiel de calcul. Il a acquis aussi une grande expérience des modèles réduits, qui, indispensables pour la solution de quelques prohlèmes (pertes de charge concentrées au pied de la cheminée d'équilibre, ete.), sont un auxiliaire précieux dans la similitude de Durand.

L'auteur tient à signaler que le présent article est le résultat non seulement de ses recherches et travaux personnels, mais aussi de l'expérience accumulce au cours des années au Laboratoire Dauphinois d'Hydraulique. Il fait aussi remarquer que s'il a repris dans son exposé deux sujets classiques comme les équations fondamentales des cheminées d'culuilibre et la similitude, c'est pour insister d'une facon toute particulière sur quelques éléments qui risquent d’induire en erreur le calculateur non averti, notamment la définition correcte de la résistance d'étranglement des cheminées d'ápuilibre munies de ce dispositif.

A sa connaissance, les méthodes proposées aux chapitres 2 et 5, références bibliographiques aux traités classiques de MM. Calane et Gaden, et Frank et Schulder exceptées, voient le jour pour la première fois.

Finalement, l'auteur souhaite que sa contribution à l'étude des cheminces d'équilibre puisse etre utile à tous ceux qui ont à s'oceuper du calcul, toujours délicat, de ces ouvrages. Il serait trìs heureux de recevoir dans les pages de cette revue toute suggestion ou communication concernant des travaux théoriques ou expérimentaux permettant d'apporter de nouvelles lumières sur cette question. 


\section{I. - LES EQUATIONS DE L'OSCILLATION EN MASSE}

Par application du théorème de Brrnovltr génernlise, a la masse d'eau contenue dans la gawrio damence, entre la retenue et le pied de la chombre d'equilibre, on obtient l'équation suivinte:

$$
\frac{1}{d f_{g}} \frac{d \mathrm{Q}_{a}}{d t}+\mathrm{Z}+\mathrm{P}+\mathrm{R}=0
$$

St hanlerie d'amence comporte plusieurs tronwon do sections $f_{g}{ }^{i}$ et longueurs $\mathrm{L}_{q}{ }^{i}$, on aura :

$$
\frac{\mathrm{L}_{g}}{f_{g}}=\Sigma \frac{\mathrm{L}_{g}^{i}}{f_{g}^{i}}
$$

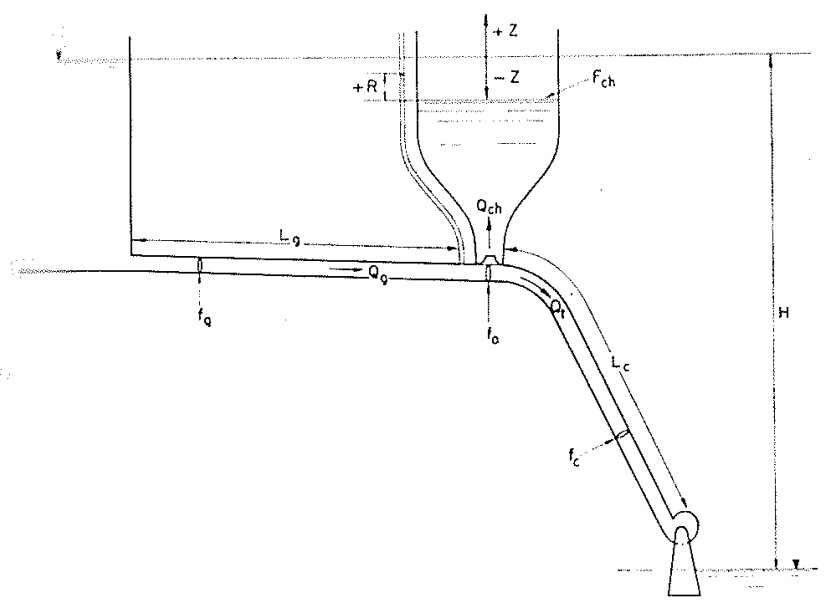

Fig. 1

$Z$ est le niveau libre dans la cheminée d'équilibre, mesuré positivement vers le haut à partir du niveau de retenue.

$P$ est la perte de charge totale dans la galerie d'amence depuis la retenue jusquau pied de la cheminée d'équilibre plus l'énergie cinctique $\mathrm{Q}, y^{2} /\left(2 g / 0^{2}\right)$.

$R$, que nous appellerons résistance d'étranglement, est la différence entre le niveau piézométrique dans la galerie d'amenée au pied de la cheminée d'équilibre et le niveau libre dans celle-ci; il est positif lorsque le niveau monte.

Pour déduire l'équation (1), les hypotheses admises sont les suivantes:

- Les parois de la galerie d'amenée sont rigides et l'eau est incompressible.

-..- L'inertie de la masse d'eau contenue dans la chambre d'équilibre est petite par rapport à celle de la galerie. L'inertie correspon- dant, pour une variation de débit donnée, au terme $\Xi(\mathbf{L} / \mathcal{f})$, cette hypothèse est exacte dans la presque totalité des cas.

-..- La répartition des vitesses dans la section $f_{0}$ est uniforme.

_- En régime varié, l'énergie cinétique des particules d'eau du niveau libre dans la cheminée est négligeable. Compte tenu des données habituelles, ceci est presque toujours vérifié.

Il est d'usage de dire que $P$ et $R$ sont respectivement proportionnels aux carrés des débits $\mathrm{Q}_{q}$ et $\mathrm{Q}_{c \pi}$.

En ce qui concerne $P$, ceci revient à admettre que l'écoulement dans la galerie est pleinement turbulent -... ce qui est admissible compte tenu des nombres de Rexnolos très élevés qu'on trouve - et que les pertes de charge en régime varié sont exprimées par les mêmes lois qu'en mouvement permanent. Cette dernière hypothèse est vérifiée d'une facon indirecte par l'accord entre les oscillations en masse calculées de la sorte et celles que l'on a mesurées sur modèle réduit, ou sur des installations réelles [1] [2].

Examinons maintenant la résistance d'étranglement. Pour les formes hydrauliques habituellement rencontrées, en régime permanent, la récupération de l'énergie cinétique de la salerie dans la chambre est presque nulle, c'est-à-dire que $R$ tel que nous l'avons défini est nul aussi. Si on prenait pour $P$ et $R$ les pertes de charge entre la retenue et le pied de la cheminée, et entre ce point et le niveau libre, l'équation (1) serait toujours valable, mais $R$ ne pourrait pas être proportionnel à $\mathrm{Q}^{2} \cdot{ }_{l l}$, car en régime permanent $\left(Q_{r}=0\right)$ il serait du même ordre de grandeur que $\mathrm{Q}_{0}^{2} /\left(2 g f_{0}^{2}\right)$. T'erreur commise serait d'autant plus importante que cette énergie cinétique est grande par rapport aux pertes de charge.

Deux autres hypothèses concernant la résistance d'étranglement sont :

- Les pertes de charge en régime varié sont les mêmes qu'en régime permanent.

- $R$ ne dépend pas de la répartition des débits au pied de la cheminée. Ceci n'est pas exact, mais c'est une approximation asser bonne. Seule une étude sur modèle réduit permet de chiffrer la variation.

A l'équation (1), on ajoutera deux autres :

- L'équation de continuité :

$$
\mathrm{Q}_{g}=\mathrm{Q}_{t}+\mathrm{Q}_{c h}=\mathrm{Q}_{t}+\mathrm{F}_{e h} \frac{d \mathrm{Z}}{d t}
$$


- La loi du débit turbiné qui, dans le cas le plus général, est de la forme :

$$
\mathrm{Q}_{t}=f\left(Z, t, \frac{d Z}{d t}, \frac{\partial Q_{t}}{\partial t}\right)
$$

On a en tout trois équations pour trois inconnues:

$$
Z, Q_{g} \text { et } Q_{t}
$$

Si la galerie d'amenée comporte $n$ cheminćes d'équilibre, on aura :

- $n$ équations du type (1), une par tronçon de galerie;
-- $n$ équations de continuité, une à chaque jonction;

- 1 équation du débit turbiné;

soit $2 n+1$ équations pour un même nombre d'inconnues.

- $n$ niveaux $Z_{i}$ dans les cheminées;

- $n$ débits $\mathrm{Q}_{g_{i}}$ dans les tronçons de galeries;

-_ le débit turbiné.

Nous allons voir comment on peut se servir de ces équations pour calculer les oscillations du plan d'eau dans les cheminées d'équilibre.

\section{II. - LES CALCULS D'AVANT-PROJET}

Soit un système avec une seule cheminée d'équilibre.

Dans un avant-projet, il s'agit d'habitude de déterminer les dimensions de la cheminée pour satisfaire aux conditions ci-après :

- Coupure totale instantanée du débit maximum;

- Ouverture instantanée de $n$ à $100 \%$ du débit maximum.

Selon les cas, $n$ peut varier entre 0 et $80 \%$. Nous allons voir quelles sont les façons de mener ces calculs assez rapidement sans toutefois perdre trop d'exactitude.

Les cheminées qu'on rencontre en général sont à section constante, avec ou sans expansions à section constante en haut et en bas. Elles peuvent être munies d'un diaphragme à leur base ou d'un déversoir.

Lorsqu'on tient compte des pertes de charge, il n'y a que deux cas qui peuvent être intégrés directement :

- La fermeture totale instantanée;

- Les manœuvres qui maintiennent constants le débit turbiné et le niveau piézométrique dans la galerie au pied de la cheminée.

Dans le premier cas, on trouve :

$$
\mathrm{Q}_{t}=0 \quad \mathrm{Q}_{g}=\mathrm{Q}_{c h}=\mathrm{F}_{c h} \frac{d Z}{d t}
$$

d'où (1) devient :

$\frac{\mathrm{L}_{q} \mathrm{~F}_{c h}}{g f_{g}} \frac{d^{2} \mathrm{Z}}{d t^{2}}+\mathrm{Z}+\left(k_{1}+k_{2}\right) \mathrm{F}_{c h}^{2}\left(\frac{d \mathrm{Z}}{d t}\right)^{2}=0$

où :

$$
\mathrm{P}=k_{1} \mathrm{Q}_{g}{ }^{2} \quad \mathrm{R}=k_{2} \mathrm{Q}^{2}{ }_{\text {ch }}
$$

équation dont la solution est de la forme :

$$
\begin{gathered}
\left(\frac{\mathrm{Q}_{\eta}}{\mathrm{Q}_{0}}\right)^{2}=\frac{\mathrm{Z}}{\mathrm{P}_{0}+\mathrm{R}_{0}}+\frac{\mathrm{L}_{t \prime} \mathrm{Q}_{0}{ }^{2}}{2 g f_{\eta} \mathrm{F}_{c h}\left(\mathrm{P}_{0}+\mathrm{R}_{0}\right)^{2}} \\
{\left[1-e^{\frac{2 y \mathrm{~F}_{c h}\left(k_{1}+k_{2}\right) f_{g}}{\mathrm{~L}_{g}}}\left(\mathrm{Z}-\mathrm{P}_{0}-\mathrm{R}_{0}\right)\right]}
\end{gathered}
$$

L’indice 0 indique les grandeurs en régime permanent.

Dans le deuxième cas, on trouve :

$$
\begin{gathered}
\mathrm{Z}+\mathrm{R}=\mathrm{H}=\mathrm{C}^{\text {te }} \quad \mathrm{Q}_{g}-\mathrm{F}_{c h} \frac{d \mathrm{Z}}{d t}==\mathrm{Q}_{t}=\mathrm{C}^{\text {te }} \\
\frac{\mathrm{L}_{g}}{g f_{g}} \frac{d \mathrm{Q}_{. \prime}}{d t}+k_{1} \mathrm{Q}_{0}{ }^{2}+\mathrm{H}=0
\end{gathered}
$$

qui est également intégrable.

Voyons maintenant les éléments dont on dispose pour calculer dans chaque cas la cheminée d'équilibre.

\section{a) Manceuvres de fermeture.}

D'habitude, on envisage les manœuvres totales.

Si la chambre est à section constante ou si elle comporte plusieurs tronçons à sections différentes mais constantes, on peut appliquer l'équation (6), qui a d'ailleurs été mise en abaques en variables réduites pour faciliter les calculs [1].

Si on cherche le maximum de $Z$, on doit annuler $\mathrm{Q}_{g}$ dans l'équation (6); l'équation qui en résulte a été représentée sous forme d'abaque en variables réduites [3] [4] [5].

$\mathrm{Si}$ la cheminée a une forme quelconque, on peut calculer l'ordre de grandeur de son volume en supposant que le niveau piézométrique à son pied reste constant [3]. Le volume trouvé sera en général inférieur au volume effectif nécessaire, l'erreur étant́ plus petite dans les chambres à étranglement inférieur, déversantes, différentielles ou munies d'une chambre d'expansion surmontant un puits de section réduite. 


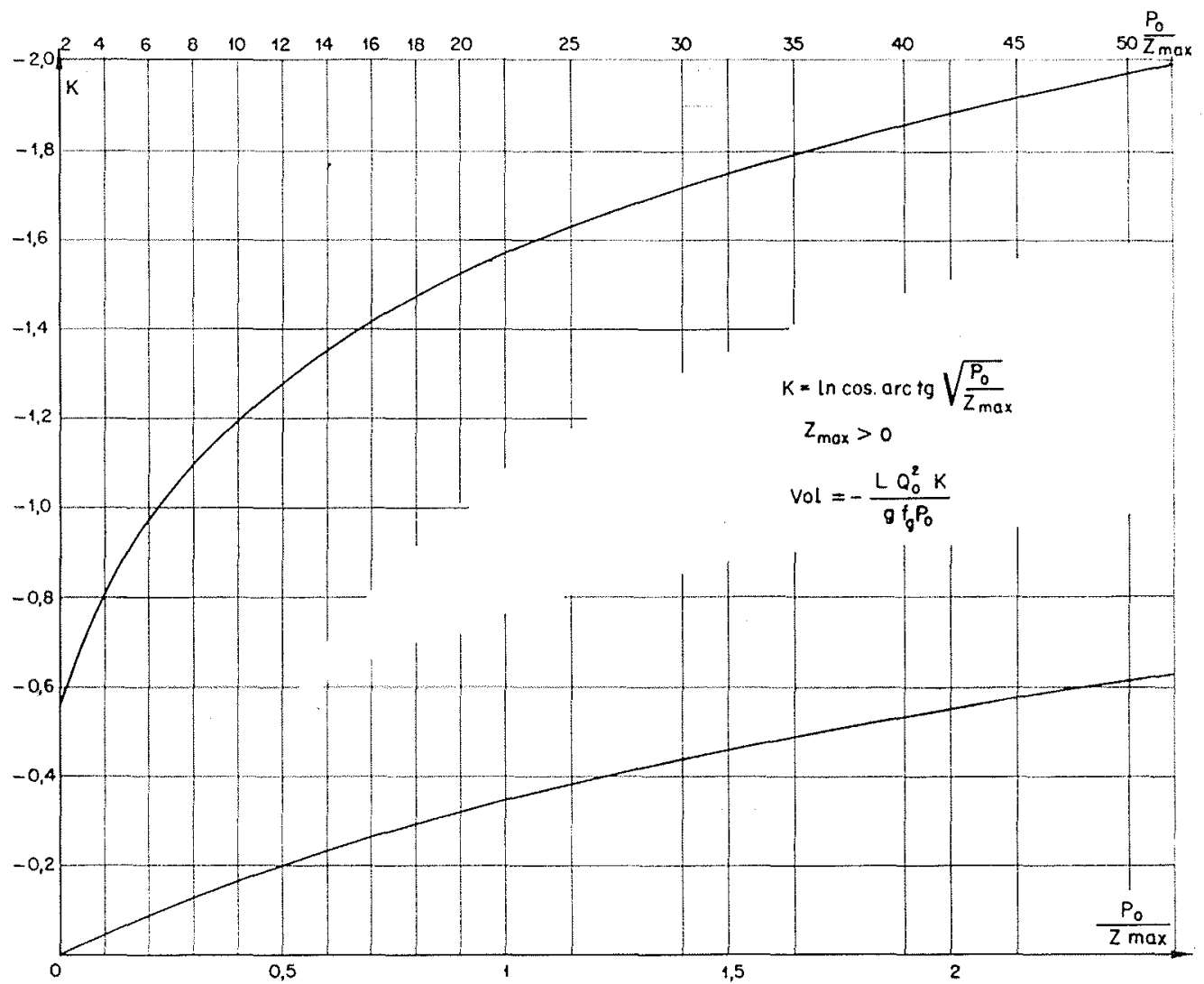

FIG. 2

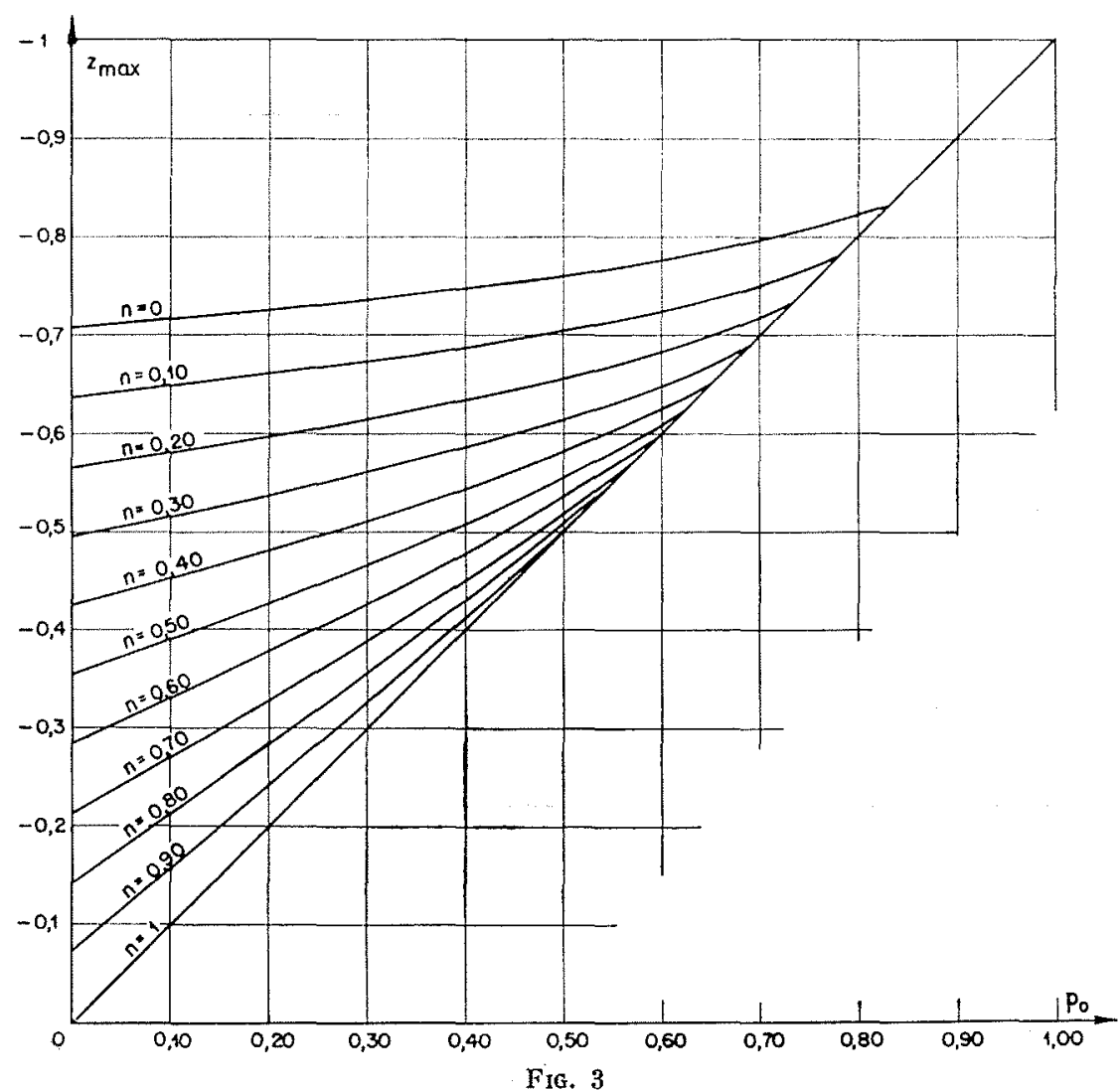


Tous calculs faits, on trouve :

vol $=\mathrm{K} \frac{\mathrm{L}_{g} \mathrm{Q}_{0^{2}}{ }^{2}}{g f_{g} \mathrm{P}_{0}^{-}} \quad$ où $\mathrm{K}=\log _{c} \cos \operatorname{arctg} \sqrt{\frac{\mathrm{P}_{0}}{\mathrm{Z}_{\mathrm{max}}}}$

La courbe de la figure 2 donne $K$ en fonction de $: \frac{P_{0}}{Z_{\max }}$

\section{b) Manceuvres̈ D'ouverture.}

Pour une ouverture instantanée de $n$ à $100 \%$ de $Q_{0}$, l'équation (7) intégrée devient:

$$
\begin{gathered}
z_{\max }=\frac{1}{2 \sqrt{-z_{\max } p_{0}}} \\
{\left[a \log _{e}\left(\frac{n-1}{a}+1\right)-b \log _{e}\left(\frac{n-1}{b}+1\right)\right]-p_{0} n^{2}}
\end{gathered}
$$

La notation est celle de MM. Calame et Gaden, c'est-à-dire :

$$
z=\frac{\mathrm{Z}}{\mathrm{Z}^{*}} \quad p_{0}=\frac{\mathrm{P}_{0}}{\mathrm{Z}^{*}} \quad \mathrm{Z} * \mathrm{Q}_{0} \sqrt{\frac{\mathrm{L}_{g}}{g f_{g} \mathrm{~F}_{c h}}}
$$

et :

$$
a=1+\sqrt{\frac{-z_{\max }}{p}} \quad b=1-\sqrt{\frac{-z_{\max }}{p}}
$$

Connaissant deux des trois paramètres $z_{\max }$, $p_{0}$ et $n$, on peut calculer le troisième avec la formule (8), ou plus rapidement avec les abaques des figures 3 et 4 , tracés d'après cette équation.

Il $\mathrm{y}$ a deux cas où la pression au pied de la cheminée se maintient presque constante au cours de la première descente et où par suite la formule peut être employée :

- Lorsque la chambre comporte un étranglement produisant, pour la manouvre envisagée, une dépression initiale égale à la descente finale;

- Lorsqu'elle a une expansion inférieure de section grande par rapport à celle du puits.

Dans le premier cas, nous aurons l'équation supplémentaire :

$$
-z_{\max }=p_{0} n^{2}+r_{0}(1-n)^{2}
$$

où :

$$
r_{0}=\frac{\mathrm{R}_{0}}{\mathrm{Z}^{*}}
$$

En connaissant trois des quatre paramètres $p_{0}, r_{0}, n, z_{\max }$, on calculera le quatrième. Les abaques des figures 5 et 6 facilitent le calcul.

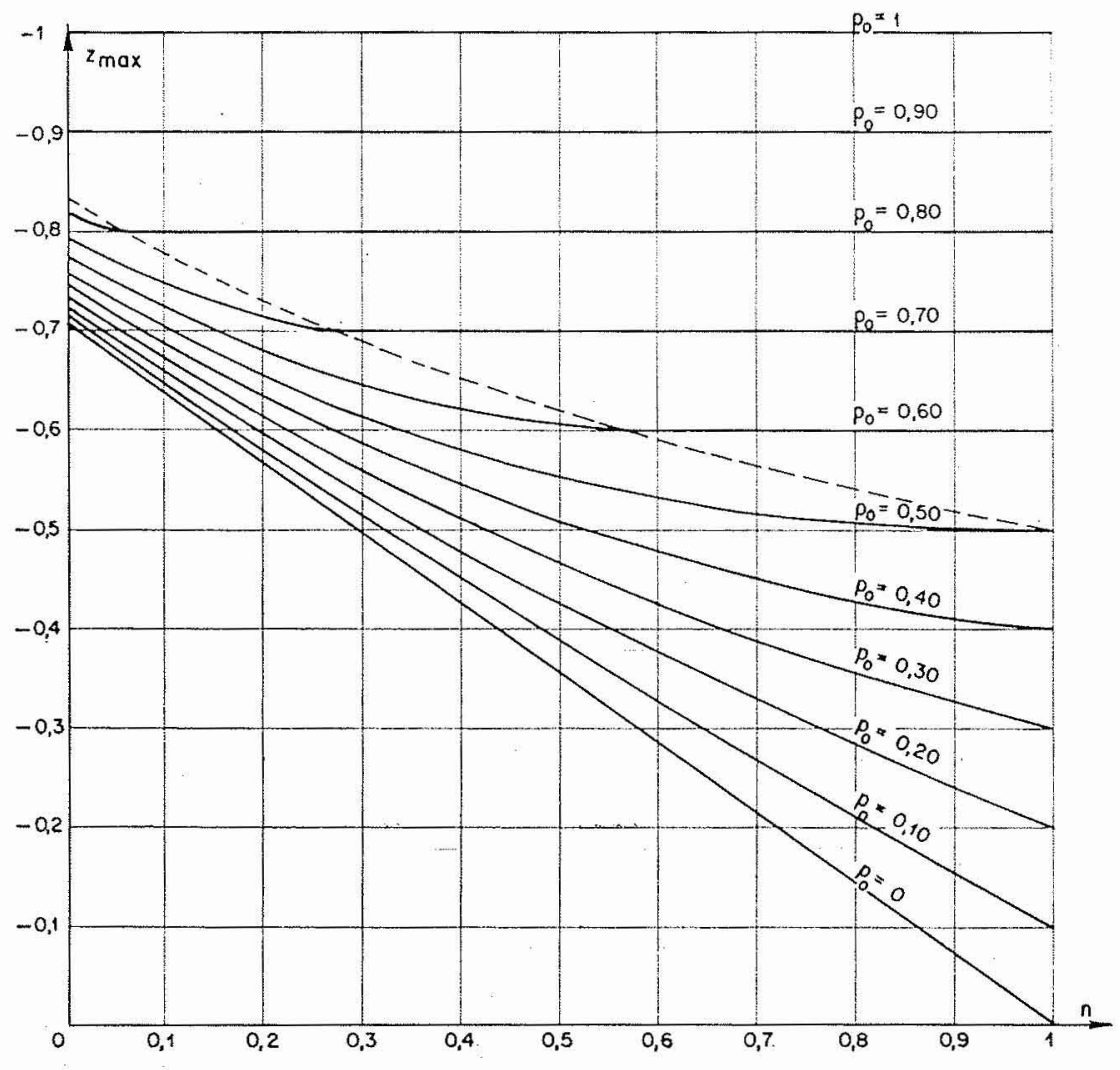

FIG. 4 


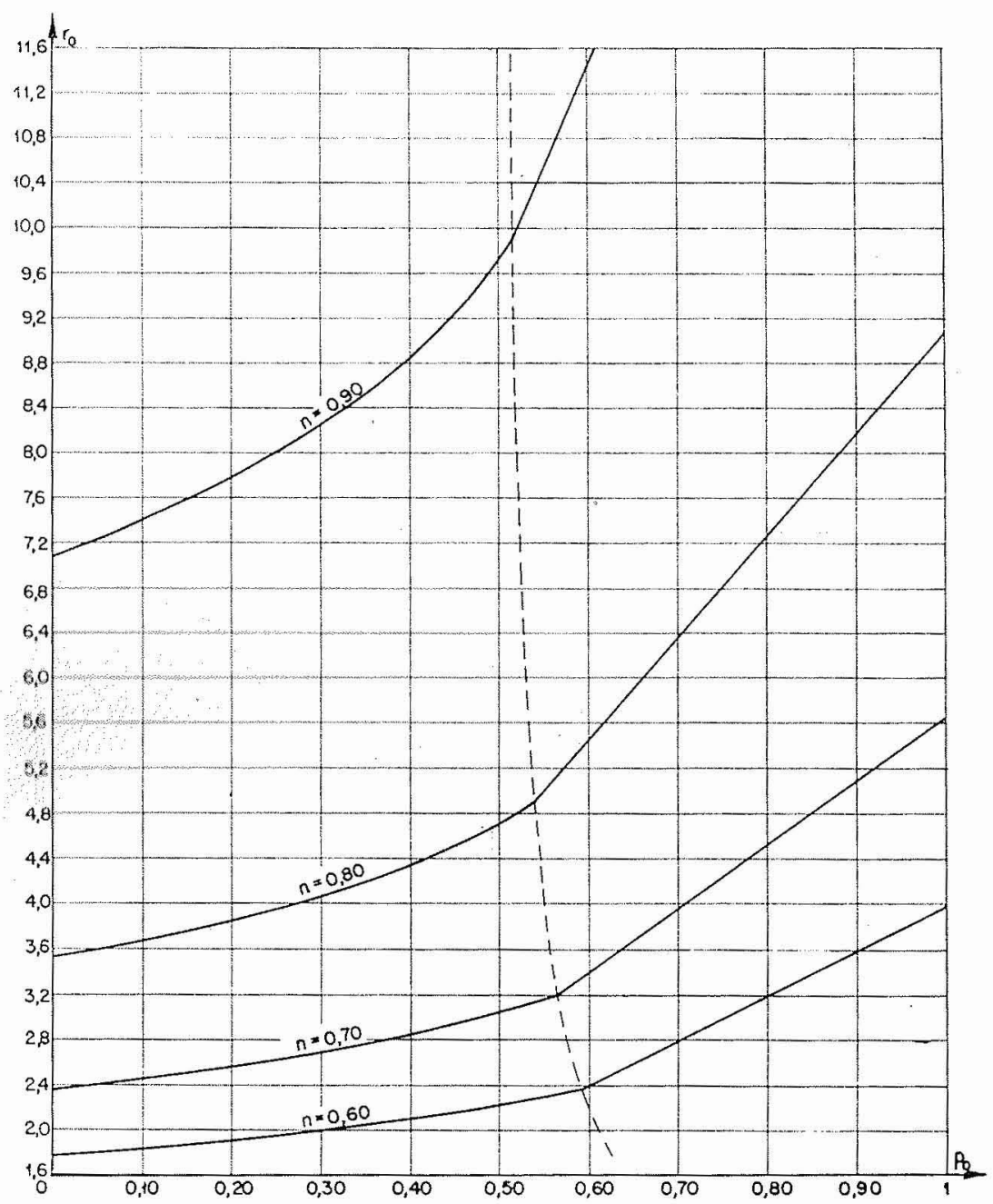

FIG. 5

\section{Exemples :}

Pour une installation ayant les caractéristiques suivantes :

$\mathrm{L}_{i j}=1.000 \mathrm{~m}$

$$
\begin{gathered}
f_{g}=10 \mathrm{~m}^{2} \\
\mathrm{P}_{0}=1 \mathrm{~m}
\end{gathered}
$$$$
\mathrm{Q}_{0}=25 \mathrm{~m}^{2} / \mathrm{s}
$$

\section{Calculer :}

$1^{\circ}$ La section de la cheminée et $\mathrm{R}$ si l'on veut pouvoir faire une prise de charge de 50 à $100 \%$ avec une descente maximum :

$$
\mathrm{Z}_{\max }=-3 \mathrm{~m} \text {. }
$$

Dans l'abaque (fig. 3), nous traçons une droite de pente:

$$
\frac{z_{\max }}{p_{0}}=\frac{Z_{\max }}{\mathrm{P}_{0}}=\left|\frac{3}{1}\right|=3
$$

Elle coupe la courbe $n=0,50$ en un point de coordonnées :

$$
p_{0}=0,133 \quad z_{\max }=-0,4
$$

d'où :

$$
\begin{aligned}
& \mathrm{F}_{c h l}=\frac{p_{0} \mathrm{Q}_{0}^{2} \mathrm{~L}_{g}}{g f_{g} \mathrm{P}_{0}}=818 \mathrm{~m}^{2} \\
& \mathrm{R}_{0}=\frac{-\mathrm{Z}_{\max }-\mathrm{P}_{0} n^{2}}{(1-n)^{2}}=11 \mathrm{~m}
\end{aligned}
$$

$2^{\circ}$ Si la section de la cheminée est de $400 \mathrm{~m}^{2}$ et la descente maximum de $2 \mathrm{~m}$, quelle est la prise de charge maximum qu'on peut faire et quelle doit être la valeur de R.

$$
\begin{gathered}
Z^{*}=25 \sqrt{\frac{1.000 !}{9,81 \times 10 \times 400}}=4,00 \mathrm{~m} \\
p_{0}=0,25 \quad z_{\max }=-0,50
\end{gathered}
$$




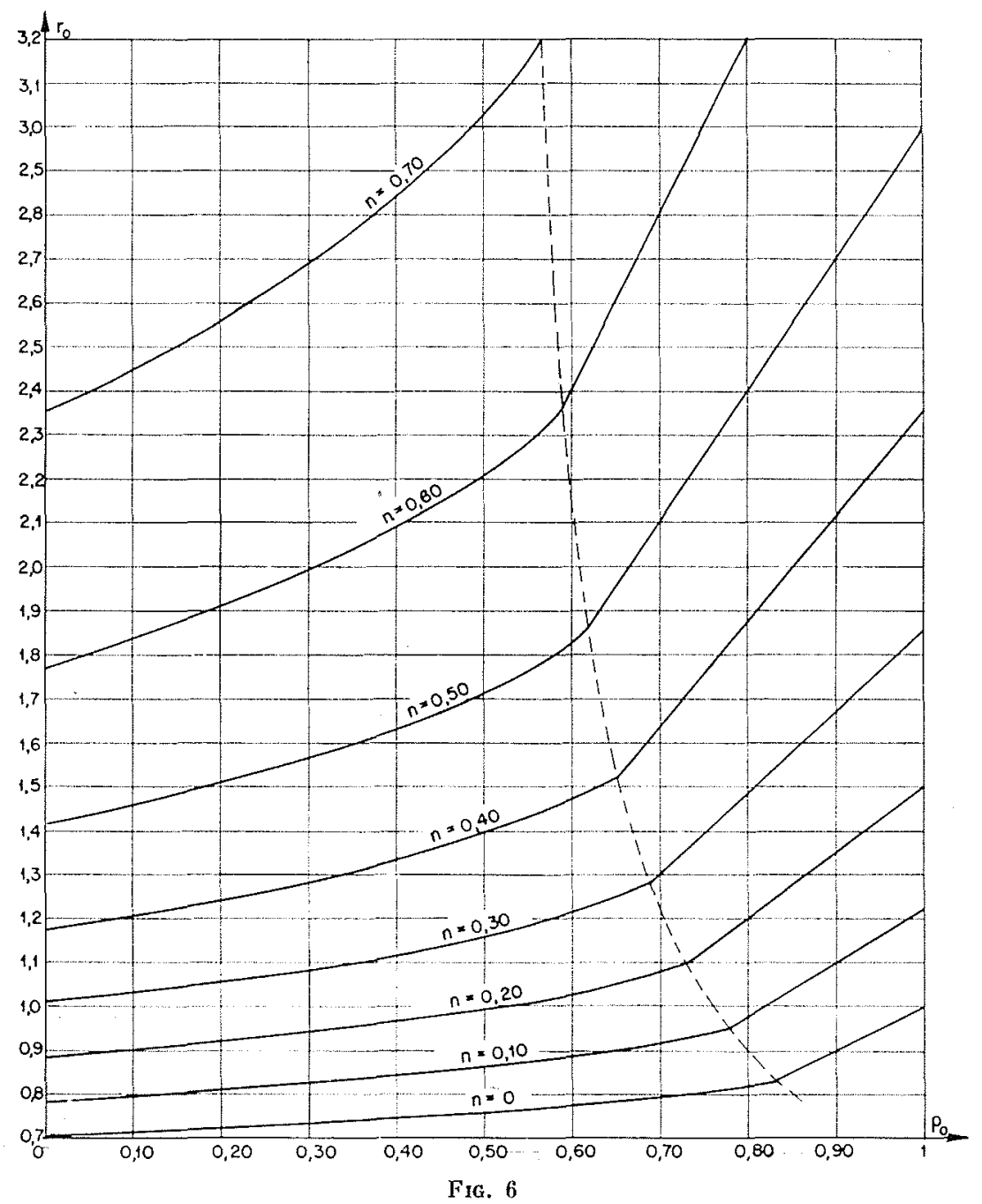

Dans la figure 4 , on trouve $n=38,5 \%$ ct avec cette valeur la figure 5 donne $r_{0}=1,23$, c'est-àd-dire :

$$
\mathrm{R}_{0}=1,23 \times 4=4,92 \mathrm{~m}
$$

Les calculs se font sans aucun tâtonnement. Tel qu'on peut le prévoir, l'expérience montre que les descentes calculées de cette facon sont légèrement inférieures à celles qu'on trouverait par un calcul plus précis. Cependant, l'erreur est faible, comme nous l'avons vérifié pour une installation ayant les caractéristiques suivantes :

$$
\begin{array}{lll}
\mathrm{L}_{\ell}=10.000 \mathrm{~m} & \mathrm{Q}_{0}=35 \mathrm{~m}^{3} / \mathrm{s} & Z_{\max }=-20 \mathrm{~m} \\
f_{o}=15 \mathrm{~m}^{2} & \mathrm{P}_{0}=13,95 \mathrm{~m} &
\end{array}
$$

Pour différents degrés d'ouverture, nous avons calculé la section de la cheminée, toujours avec un étranglement donnant une dépression initiale égale à la descente finale. Le tableau ci-après donne les résultats obtenus avec les abaques et par intégration graphique:

\begin{tabular}{|c|c|c|c|c|c|}
\hline$n \%$ & 0 & 20 & 40 & 60 & 80 \\
\hline Intégration & 125 & 96 & 62 & 41 & 19,8 \\
\hline Abaques... & 120 & 86,2 & 58,2 & 37 & 15,6 \\
\hline Erreur (\%) & 4 & 10,2 & 6,2 & 9,8 & 21 \\
\hline
\end{tabular}

On voit que, pour un premier calcul, le résultat est assez bon, les grandes valeurs de $n$ exceptées 


\section{III. - LA METTHODE DE LINEARISATION}

81. on considère les eheminées d'équilibre à whanglement inférieur, lorsque, au début de la manouve, la pression dans la galerie au pied de In chambre est différente du nivenu atteint à la Wh de la première descente (ou montée), l'équahon du mouvement n'est pas intégrable, à l'exexplion du cas de la coupure totale instantanée.

Si on tient compte de (3), l'équation (i) devient :

$$
\begin{aligned}
& \frac{\mathrm{L}_{g} \mathrm{~F}_{c h}}{g f_{g}} \frac{d^{2} Z}{d t^{2}}+Z+k_{1} \mathrm{Q}_{t^{2}}+k_{1} \mathrm{~F}_{c \mathrm{~h}}^{2}\left(\frac{d Z}{d t}\right)^{2} \\
& +2 k_{\mathrm{L}} \mathrm{Q}_{t} \mathrm{~F}_{c h} \frac{d Z}{d t}+k_{2} \mathrm{~F}_{c h}^{2}\left(\frac{d Z}{d t}\right)^{2}=0
\end{aligned}
$$

Avec le changement de variable:

$$
\mathrm{Y}=\mathrm{Z}+\mathrm{P}_{0}
$$

et si on considere que le débit turbiné est égal a $\mathrm{Q}_{0}$ tout le long de l'oscillation, on a :

$$
\begin{gathered}
\frac{\mathrm{L}_{g} \mathrm{~F}_{o h}}{g f_{g}} \frac{d^{2} \mathrm{Y}}{d t^{2}}+\left(k_{1}+k_{2}\right) \mathrm{F}_{e h}^{2}\left(\frac{d \mathrm{Y}}{d t}\right)^{2} \\
+2 k_{1} \mathrm{Q}_{0} \mathrm{~F}_{c h} \frac{d \mathrm{Y}}{d t}+\mathrm{Y}=0
\end{gathered}
$$

Cette équation n'est pas intégrable, mais elle le serait si le deuxième terme était proportionnel à $d \mathrm{Y} / d t$ au lieu de l'être à $(d \mathrm{Y} / d t)^{2}$. Si nous remplacons $\left(k_{1}+k_{2}\right) \mathrm{F}^{2} \cdot \mathrm{h}(d \mathrm{Y} / d t)^{2}$ par $\mathrm{K} d \mathrm{Y} / d t$, $K$ étant choisi de façon à rendre minime l'erreur commise le long de la première desccnte (ou montée) par la théorie des moindres carrés, nous trouvons:

$\frac{d}{d \mathrm{~K}} \int_{1}^{2}\left[\left(k_{1}+k_{2}\right) \mathrm{F}_{c h}^{2}\left(\frac{d \mathrm{Y}}{d t}\right)^{2}-\mathrm{K} \frac{d \mathrm{Y}}{d t}\right] d \frac{d \mathrm{Y}}{d t}=0$

d'où, tous calculs faits :

$$
\mathrm{K}=\frac{3}{4}\left(k_{1}+k_{2}\right) \mathrm{F}_{c h}^{2} \frac{\left(\frac{d \mathrm{Y}}{d t}\right)_{2}^{4}-\left(\frac{d \mathrm{Y}}{d t}\right)_{1}^{4}}{\left(\frac{d \mathrm{Y}}{d t}\right)_{2}^{3}-\left(\frac{d \mathrm{Y}}{d t}\right)_{1}^{3}}
$$

Pour le cas d'une ouverture de $n$ à $100 \%$ :

$$
\begin{aligned}
\left|\frac{d \mathrm{Y}}{d t}\right|_{t} & =-\frac{(1-n) \mathrm{Q}_{0}}{\mathrm{~F}_{c h}} \quad\left|\frac{d \mathrm{Y}}{d t}\right|_{2}=0 \\
\mathrm{~K} & =\frac{3}{4}\left(k_{1}+k_{2}\right)(n-1) \mathrm{Q}_{0} \mathrm{~F}_{c h}
\end{aligned}
$$

En remplaçant dans (10):

$$
\begin{gathered}
\frac{\mathrm{L}_{g} \mathrm{~F}_{c h}}{g f_{g}} \frac{d^{2} \mathrm{Y}}{d t^{2}} \\
+\left[\frac{3}{4}\left(k_{1}+k_{2}\right)(n-1) \mathrm{Q}_{0} \mathrm{~F}_{c h}+2 k_{1} \mathrm{Q}_{0} \mathrm{~F}_{c h}\right] \frac{d \mathrm{Y}}{d t} \\
+\mathrm{Y}=0
\end{gathered}
$$

ou, d'une façon symbolique :

$$
\mathrm{A} \ddot{\mathrm{Y}}+\mathrm{B} \dot{\mathrm{Y}}+\mathrm{Y}=0
$$

L'équation caractéristique est :

$$
\mathrm{A} r^{2}+\mathrm{Br}+1=0
$$

\section{Discussion.}

$$
\text { a) } \mathrm{B}^{2}>4 \mathrm{~A} \text {. }
$$

Le mouvement sera apériodique et l'on passera sans oscillation de l'ancien au nouveau niveau piézométrique.

En remplaçant A et B par leurs valeurs, on trouve :

$$
\mathrm{R}_{0} \geq \frac{8}{3} \frac{1}{1-n}\left(\mathrm{P}_{0}-\mathrm{Z}^{*}\right)-\mathrm{P}_{0}
$$

On n'a aucun intérèt à augmenter $R_{0}$ au-delà de cette valeur limite car la descente maximum sera toujours la même.

Cette formule a été appliquée à la cheminée d'équilibre de Rio Tercero II (Argentine), installation qui avait les données suivantes :

$$
\begin{array}{ll}
\mathrm{L}_{g}=873 \mathrm{~m} . & \mathrm{Q}_{0}=52,5 \mathrm{~m} / \mathrm{s} \\
f_{g}=19,03 \mathrm{~m}^{2} . & \mathrm{F}_{c h}=191 \mathrm{~m}^{2} . \\
\mathrm{P}_{0}=2,38 \mathrm{~m} . & n=0,7 .
\end{array}
$$

La formule (17) a donné $\mathrm{R}_{0}=54,20 \mathrm{~m}$, ce qui a permis de dégrossir très rapidement le problème, car on cherchait une descente oscillatoire mais pas très éloignée de l'apériodicité. Par intégration graphique, on a trouvé :

b) $\mathrm{B}^{2}<4 \mathrm{~A}$.

$$
Z_{\max }=--2,90 \mathrm{~m}, \quad \text { pour : } R_{0}=46 \mathrm{~m}
$$

Le mouvement oscillatoire répond à l'équation :

$$
\mathrm{Y}=e^{-(\mathrm{B} / 2 \mathrm{~A}) t}
$$

$\left[M \sin \sqrt{4 \pi^{2}-\frac{\mathrm{B}^{2}}{4 \mathrm{~A}^{2}}} t+\mathrm{N} \cos \sqrt{4 \pi^{2}-\frac{\mathrm{B}^{2}}{4 \mathrm{~A}^{2}}} t\right]$ 


$$
\begin{aligned}
\mathrm{Y}= & e^{-(\mathrm{P} / 2 \mathrm{~A}) t}\left[-\frac{\mathrm{BM}}{2 \mathrm{~A}} \sin \sqrt{4 \pi^{2}-\frac{\mathrm{B}^{2}}{4 \mathrm{~A}^{2}}} t\right. \\
& -\frac{\mathrm{BN}}{2 \mathrm{~A}} \cos \sqrt{4 \pi^{2}-\frac{\mathrm{B}^{2}}{4 \mathrm{~A}^{2}}} t \\
& +\mathrm{M} \sqrt{4 \pi^{2}-\frac{\mathrm{B}^{2}}{4 \mathrm{~A}^{2}}} \cos \sqrt{4 \pi^{2}-\frac{\mathrm{B}^{2}}{4 \mathrm{~A}^{2}}} t \\
-\mathrm{N} & \sqrt{4 \pi^{2}-\frac{\mathrm{B}^{2}}{4 \mathrm{~A}^{2}}} \sin \sqrt{4 \pi^{2}-\frac{\mathrm{B}^{2}}{4 \mathrm{~A}^{2}}} t
\end{aligned}
$$

$\mathrm{M}$ et $\mathrm{N}$ étant les constantes d'intégration.

$$
\begin{aligned}
& \text { Pour : } t=0, \\
& \mathrm{Y}=k_{1} \mathrm{Q}_{0}^{2}\left(1-n^{2}\right) \quad \frac{d \mathrm{Y}}{d t}=\frac{\mathrm{Q}_{0}(n-1)}{\mathrm{F}_{c h}}
\end{aligned}
$$

d'où :

$$
\mathrm{N}=k_{1} \mathrm{Q}_{0}{ }^{2}\left(1-n^{2}\right)
$$

Si on appelle :

$$
\mathrm{C}=\frac{\mathrm{B}}{2 \mathrm{~A}}=\frac{\left[\frac{3}{4}\left(k_{1}+k_{2}\right)(n-1)+2 k_{1}\right\rfloor g \mathrm{Q}_{0} f_{g}}{2 \mathrm{~L}_{g}}
$$

On trouve :

$$
\mathrm{M}=\frac{\frac{\mathrm{Q}_{0}}{\mathrm{~F}_{c h}}(n-1)+\mathrm{C} k_{1} \mathrm{Q}_{0}{ }^{2}\left(1-n^{2}\right)}{\sqrt{4 \pi^{2}-\mathrm{C}^{2}}}
$$

Avec $\mathrm{M}$ et $\mathrm{N}$, en partant de l'équation (19), on calcule le temps $t_{1}$ an bout duquel on a $\mathrm{Y}=\mathrm{Y}_{\min }$ et $d \mathrm{Y} / d t=0$.

$$
t_{1}=\frac{1}{\sqrt{4 \pi^{2}-\mathrm{C}^{2}}} \operatorname{arctg} \frac{\mathrm{CN}+\mathrm{M} \sqrt{4 \pi^{2}-\mathrm{C}^{2}}}{\mathrm{CM}+\mathrm{N} \sqrt{4 \pi^{2}-\mathrm{C}^{2}}}
$$

Introduite dans l'équation (18), cette valeur de $t_{1}$ permet de calculer la descente maximum :

$$
\mathrm{Z}_{\max }=\mathrm{Y}_{\max }-\mathrm{P}_{0}
$$

Les calculs d'ouverture fractionnaire faits avec cette période et vérifiés par intégration graphique ont donné une erreur de quelques centièmes, c'est-à-dire du même ordre de grandeur que la précision de l'épure.

\section{IV. - CALCUL DÉfINITIF DES DIMENSIONS DE LA CHAMBRE D'EQUILIBRE}

Une fois fixées, par un calcul simplifié, il faut vérifier que les dimensions de la chambre d'équilibre sont suffisantes pour assurer la bonne marche de l'installation, compte tenu des conditions effectives d'exploitation les plus sévères.

Pour cela, il faut faire, dans chaque cas, une intégration pas à pas des équations (1), (3) et (4). Parmi les diverses méthodes graphiques, nous avons retenu celle qui a été proposée par M. Schoklitsch [6] en 1923, pour être reprise et complétée plus tard par M. Schiffmann [7], Del Felice [8], et dernièrement par MM. BouVARD et Molbert [9], qui, dans un exposé remarquable, ont mis en relief les vastes possibilités de cette méthode, même dans des cas extrêmement complexes.

Pour les chambres d'équilibre à étranglement, il est très important de vérifier les manœuvres les plus défavorables en se rappelant que, pour un débit donné, celles-ci ne sont pas forcément les manœuvres brusques du plus grand débit. Ainsi, déjà dans les abaques de MM. Calame et GADEN [4], on voit que si, pour une installation donnée, $\mathrm{R}_{0}$ est suffisamment grand par rapport à $\mathrm{P}_{0}$, la coupure d'un débit inférieur au maximum peut donner une montée plus grande; ceci s'explique par le fait que le débit coupé étant plus petit, l'action de freinage de l'étranglement proportionnelle à son carré sera plus réduite; quẹlquefois ce facteur étant prédominant, la montée est plus grande. Un procédé graphique, basé sur la méthode SchoкLiтsch, a été mis au point au Laboratoire Dauphinois d'Hydraulique, pour permettre de trouver en même temps la manœuvre la plus défavorable et la montée qui en résulte. Son principe est le suivant : si, à partir d'un état quelconque au temps $t_{0}$ et pendant un intervalle de durée $\Delta t$, nous faisons varier de $\left(d \mathrm{Q}_{t} / d t\right)_{0} \Delta t$ le débit turbiné, il $\mathrm{y}$ aura pendant ce même intervalle des variations $(d Z / d t)_{0} \Delta t$ et $\left(d Q_{g} / d t\right)_{0} \Delta t$ du niveau dans la cheminée et du débit dans la galerie d'amenée. II est évident que pour une valeur particulière de $\left(d Q_{t} / d t\right)_{0}$, le rapport :

$$
\frac{\left(\frac{d Z}{d t}\right)_{0} \Delta t}{\left(\frac{d Q_{g}}{d t}\right)_{0} \Delta t}=\left(\frac{d Z}{d Q_{g}}\right)_{0}
$$

sera maximum. Cette manœuvre élémentaire sera la plus défavorable que l'on puisse faire au temps $t_{0}$; de plus, l'opération la plus défavora- 
ble possible, à partir d'un état initial quelconque, sera constituée par une série de manœuvres élémentaires telles qu'à chaque instant on vérifie que :

$$
\frac{\partial}{\partial Q_{t}} \frac{d Z}{d Q_{t}}=0
$$

Nous allons mettre cetie équation sous forme explicite. De (1) ot (3), on déduit que :

$$
\frac{d \mathrm{Q}_{t}}{d t}=-\frac{g f_{l}}{\mathrm{~L}_{g}}\left[\mathrm{Z}+k_{1} \mathrm{Q}_{g^{2}}+k_{2} \mathrm{~F}_{c \cdot h}^{2}\left(\frac{d Z}{d t}\right)^{2}\right] \text { et } \frac{d \mathrm{Z}}{d t}=\frac{1}{\mathrm{~F}_{c h t}}\left(\mathrm{Q}_{t}-\mathrm{Q}_{t}\right)
$$

d’ò̀ :

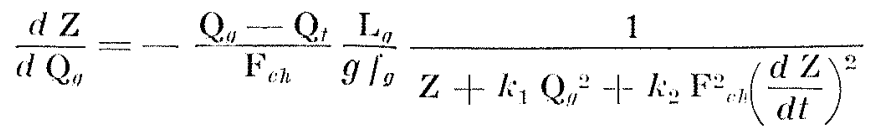

$$
\begin{aligned}
& \frac{\partial}{\partial Q_{t}} \frac{d Z}{d Q_{t}}=\frac{\mathrm{L}_{l}}{g \mathrm{~F}_{c h} t_{l}} \frac{Z+k_{1} Q_{t^{2}}+k_{2} \mathrm{~F}_{c h}^{2}\left(\frac{d \mathrm{Z}}{d t}\right)^{2}-\left(\mathrm{Q}_{g}-\mathrm{Q}_{t}\right)^{2} 2 k_{2}}{\left[\mathrm{Z}+k_{1} \mathrm{Q}_{t^{2}}+k_{2} \mathrm{~F}^{2}{ }_{c h}\left(\frac{d \mathrm{Z}}{d t}\right)^{2}\right]^{2}}=0
\end{aligned}
$$

d'où :

$Z+k_{1} Q_{t}+k_{n} \mathrm{~F}_{0}\left(\frac{d Z}{d t}\right)^{2}-2 k_{2} \mathrm{~F}^{2}\left(\frac{d Z}{d t}\right)^{2}=0$

60 qui sevient at :

$$
Z+P=R
$$

Cette condition est extrêmement simple, surtout lorsqu'il s'agit de faire une épure SchokurTsch, dans laquelle on porte $Z$ et $Q_{g}$, respectivement en ordonnées et en abscisses. La parabole $\mathrm{P}=k_{1} \mathrm{Q}_{g}{ }^{2}$ étant tracée, la distance verticale du point représentatif de l'instant $t_{0}$, à la

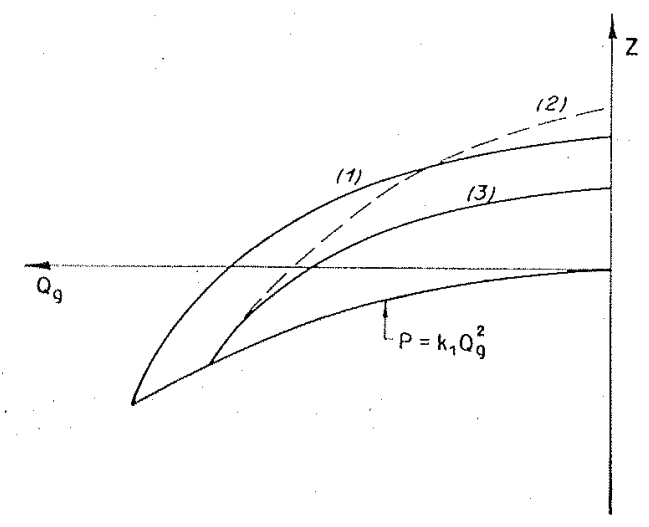

Fig. 7 parabole $\mathrm{P}$ donne la valeur instantanée de $\mathrm{R}$, qui permet de connaitre $Q_{r h}$ et de tracer la variation élémentaire de niveau pendant l'intervalle $\Delta t$.

La manœuvre la plus défavorable est celle qui met en jeu le plus grand débit. En effet, dans les figures 7 et 8 , les courbes (1) et (3) représentent des manœuvres du type que nous venons d'expliquer, l'une de fermeture, l'autre d'ouver. ture, pour deux débits différents. Toute courbe de la forme (2) ne serait pas possible, car elle couperait la courbe (1) avec une tangente plus grande, ce qui est contraire à la définition même de la courbe (1) qui a, en tous ses points, la dérivée maximum.

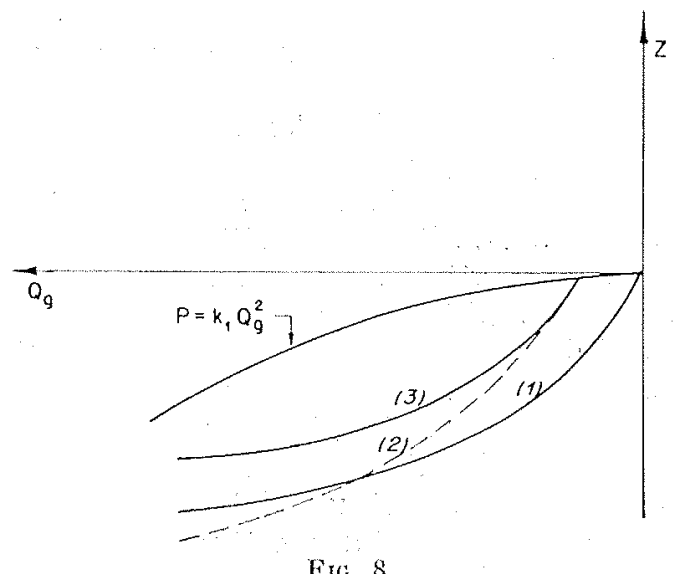

\section{V. - LA STABILITE DES OSCILLATIONS EN MASSE}

La formule classique de Troma:

$$
\mathrm{F}_{t, h}=\frac{\mathrm{Q}_{0}^{2}}{2 g} \frac{\mathrm{L}_{g}}{f_{g}\left(\mathrm{H}-\Delta \mathrm{H}_{g}\right) \Delta \mathrm{H}_{q}}
$$

où $\Delta \mathrm{H}_{l,}$ est la perfe de charge pour le débit $\mathrm{Q}_{0}$ dans la galerie d'amenée, donne la section mi- nimum de la cheminée d'équilibre assurant la stabilite des oscillations infiniment petites autour d'un etat d'equilibre lorsque les turbines fournissent une puissance constante.

Dans cette formule, on néglige l'action des conduites forcées (inertie et pertes de charge), 
l'énergie cinétique dans la galerie au pied de la cheminée, le statisme, l'action des autres centrales débitant en parallèle, les variations de rendement des turbines et les recouvrements et jeux du régulateur.

Divers auteurs ont complété la formule de 'Troma en évaluant l'action de ces différents termes [10] [11].

Par exemple, si l'on tient compte de l'inertic et de la perte de charge dans la conduite forcée, ainsi que de l'énergie cinétique $\mathrm{Q}_{0}{ }^{2} /\left(2 g f_{0}{ }^{2}\right)$, on trouve :

$\mathrm{F}_{\text {miu }}=\frac{\mathrm{Q}_{0}^{2}}{2 g} \frac{\frac{\mathrm{L}_{l}}{g f_{t \prime}}+\frac{\mathrm{L}_{c}}{f_{c}}}{\left(\mathrm{H}-\Delta \mathrm{H}_{g}+\frac{\mathrm{Q}_{0}^{2}}{g f_{0}^{2}}-3 \Delta \mathrm{H}_{c}\right)\left(\Delta \mathrm{H}_{l t}+\frac{\mathrm{Q}_{0}{ }^{2}}{2 g f_{0}{ }^{2}}\right)}$

où l'indice $c$ se rapporte aux éléments de la conduite forcée.

Ces formules donnent une bonne sécurité malgré la simplicité de leur schéma de calcul, car depuis 1910, date à la quelle Тнома émit sa théorie à la suite des instabilités enregistrées à la Centrale de Heimbach, on a rarement entendu parler de ce phénomène. Des essais faits en Italic au cours de ces dernières années [12], [13], montrent qu'on aurait tout intérêt à serrer d'aussi près que possible les calculs à la réalité pour être sûr que ces formules n'impliquent pas une sécurité excessive.

Il est d'autre part logique que les formules de la forme (27) ou (28) ne donnent pas une idée très nette de la stabilité, car si le schéma de calcul qui permet de les déduire repose presque toujours sur l'hypothèse d'oscillations infiniment petites, en pratique le problème se pose d'une autre façon : si l'installation a une certaine tendance à l'instabilité, les oscillations augmenteront jusqu'à atteindre au bout d'un certain temps une amplitude limite sur laquelle les formules ci-dessus ne donnent aucune indication.

Pour essayer de chiffrer, au moins dans une première approximation, la valeur de l'amplitude limite, nous admettrons qu'à la fin les oscillations ont une allure sinusoïdale. En faisant cela, nous nous inspirons d'une méthode proposée par M. Johnson [14] pour les cheminées différentielles. M. JaEGER [15] a admis aussi cette hypothèse dans une recherche portant cependant sur un but différent.

Si on admet qu'au régime final les oscillations entretenues'sont de la forme :

$$
\mathrm{Y}=\mathrm{Z}+\mathrm{P}_{0}=\mathrm{Y}_{l} \sin \frac{2 \pi t}{\mathrm{~T}}
$$

où :

$$
\mathbf{T}=2 \pi \sqrt{\frac{\mathbf{L}_{g} \mathbf{F}_{r h}}{g f_{g}}}
$$

on ne cherchera pas à vérifier les équations différentielles à tout instant, mais leur intégrale sur une demi-période, comptée à partir d'un niveau extrême dans la cheminée d'équilibre. Cette hypothèse n'est qu'une première approximation, mais elle a le mérite de la simplicité des calculs.

Si on néglige l'influence des conduites forcées, la récupération de l'énergie cinétique au pied de la cheminée et les variations de rendement en fonction du débit et de la charge, on a :

$$
\mathrm{Q}_{t}(\mathrm{H}+\mathrm{Z}+\mathrm{R})=\mathrm{Q}_{0}\left(\mathrm{H}-\mathrm{P}_{0}\right)=\mathrm{Q}_{0} \mathrm{H}_{0}
$$

d'où :

$$
\frac{Q_{t}}{Q_{0}}=\frac{\mathrm{H}_{0}}{\mathrm{H}_{0}+\mathrm{Y}+\mathrm{R}}=\frac{\mathrm{H}_{0}}{\mathrm{H}_{0}+\mathrm{M}}
$$

$\mathrm{Si}$ nous adoptons les paramètres adimensionnels de MM. Galame et Gaden :

$$
\begin{gathered}
v=\frac{\mathrm{Q}_{c h}}{\mathrm{Q}_{0}} \quad u=\frac{\mathrm{Q}_{t}}{\mathrm{Q}_{0}} \quad w=\frac{\mathrm{Q}_{g}}{\mathrm{Q}_{0}} \\
p=\frac{\mathrm{P}}{\mathrm{Z}^{*}}=p_{0} w^{2} \quad r=\frac{\mathrm{R}}{Z^{*}}=r_{0} \nu^{2} \quad h_{0}=\frac{\mathrm{H}_{0}}{Z^{*}} \\
y=\frac{\mathrm{Y}}{Z^{*}} \quad m=\frac{\mathrm{M}}{Z^{*}} \quad t^{\prime}=\frac{t}{\mathrm{~T}}
\end{gathered}
$$

Les équations (1), (3), (29) et (30) deviennent :

$$
\begin{aligned}
& \quad \frac{1}{2 \pi} \frac{d w}{d t^{\prime}}+y+p_{0}\left(w^{2}-1\right)+r_{0} v^{2}=0 \\
& w=u+v \quad(33) \quad y=y_{1} \sin 2 \pi t^{\prime} \\
& u=\frac{h_{0}}{h_{0}+m}=1-\frac{m}{h_{0}}+\frac{m^{2}}{h_{0}^{2}}-\ldots
\end{aligned}
$$

Si les oscillations sont finies, mais petites par rapport à $\mathrm{H}_{0}$, on négligera les termes d'ordre supérieur à $\left(m / h_{0}\right)^{2}$, d'où :

$$
\begin{gathered}
u=1-\frac{m}{h_{0}}+\left(\frac{m}{h_{0}}\right)^{2} \\
=1-\frac{y}{h_{0}}-\frac{r}{h_{0}}+\frac{y^{2}}{h_{0}{ }^{2}}+\frac{r^{2}}{h_{0}{ }^{2}}+\frac{2 r y}{h_{0}{ }^{2}}
\end{gathered}
$$

Si on intègre (32) entre $t_{0}=-1 / 2$ et $t_{1}=1 / 2$ et que l'on néglige les puissances de $y_{l}$ de degré supérieur au carré, tous calculs faits on trouve :

$$
\begin{gathered}
\frac{2}{3 \pi} \frac{p_{0}}{h_{0}}\left(2 r_{0} \pm \frac{1}{h_{0}}\right) y_{l}^{2} \pm \\
\left(\frac{p_{0}+r_{0}}{4}-\frac{p_{0} r_{0}}{2 h_{0}} \mp \frac{3 p_{0}}{4 h_{0}^{2}}\right) y_{l}+\frac{1-2 p_{0} h_{0}}{\pi h_{0}}=0
\end{gathered}
$$

Le signe + ou - varie selon que l'intégration part du niveau maximum ou du niveau minimum. 
Nous simplifierons cetle équation pour la transformer en :

$$
\frac{4}{3 \pi} \frac{p_{0} r_{0}}{h_{0}} y_{l}^{2} \pm\left(\frac{p_{0}+r_{0}}{4}-\frac{p_{0} r_{0}}{2 h_{0}}\right) y_{l}+\frac{1-2 p_{0} h_{0}}{\pi h_{0}}=0
$$

veci est en général possible car :

$$
\frac{3 p_{0}}{4 h_{0}{ }^{2}} \text { est petit par rapport à } \frac{p_{0}+r_{0}}{4}-\frac{p_{0} r_{0}}{2 h} \text {, et } 2 r_{0} \text { est en général grand par rapport à }-\frac{1}{h_{0}} \text {. }
$$

S'il n'en était pas ainsi, le coefficient deviendrait petit et $(36$ bis $)$ domerait:

$$
\eta_{l}=\frac{1-2 p_{0} h_{0}}{\pi h_{0}\left(\frac{p_{0}+r_{0}}{4}-\frac{p_{0} r_{0}}{2 h_{0}}\right)}
$$

$$
y_{l}= \pm \frac{\left(\frac{p_{0}+r_{0}}{4}-\frac{p_{0} r_{0}}{2 h_{0}}\right) \pm \sqrt{\left(\frac{p_{0}+r_{0}}{4}-\frac{p_{0} r_{0}}{2 h_{0}}\right)^{2}-\frac{16}{3 \pi^{2}} \frac{p_{0} r_{0}}{h_{0}{ }^{2}}\left(1-2 p_{0} h_{0}\right)}}{\frac{4}{3 \pi} \frac{p_{0} r_{0}}{h_{0}}}
$$

Dans cette équation, il laut toujours prendre la plus petite valeur de $! / \%$.

Notoas que 1 - $2 p_{0} h_{0}$ est l'expression, en varinbles reduites, de la condition de Thoma (27). Si clle est verifte, $(38)$ devient $y_{l}=0$.

Dans le cas particulier, où l'on peut négliger $p_{0}$ ou $x_{0}$, on $a$ :

$$
\begin{array}{ll}
p_{0}=0 & y_{l}=\frac{1,27}{r_{0} h_{0}} \\
r_{0}=0 & y_{l}=1,27 \frac{1-2 p_{0} h_{0}}{p_{0} h_{0}}
\end{array}
$$

Notons que M. Escande [16] a proposé la formule :

$$
y_{t}=1,18 \frac{1-2 p_{0} h_{0}}{r_{0}\left(h_{0}-2 p_{0}\right)}
$$

Si on considère les deux cas limites ci-dessus, on trouve :

$$
\begin{array}{ll}
p_{0}=0 & y_{l}=\frac{1,18}{r_{0} h_{0}} \\
r_{0}=0 & y_{l}=\infty
\end{array}
$$

Lorsque $r_{0} \rightarrow 0$, la formule (37) donne encore des résultats finis, tandis que celle de M. EsCande fait croitre l'amplitude $y_{l}$ au-dela de toute limite.

Pour vérifier le degré d'approximation de la formule (40), nous l'avons appliquée aux minutieux essais de stabilité faits par M. GHertr à la Centrale de Partidor [13] qui était munie, pour

\begin{tabular}{|c|c|c|c|c|}
\hline \multirow[b]{2}{*}{$\vec{\pi}$} & \multirow{2}{*}{ 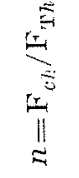 } & \multicolumn{2}{|c|}{$\mathrm{Y}_{1}$} & \multirow[b]{2}{*}{ Observations } \\
\hline & & 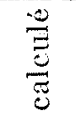 & 晜 & \\
\hline $7 b$ & 0,758 & 0,68 & 0,95 & $\begin{array}{l}\text { Au moment de la mesure les } \\
\text { oscillations étaient encore } \\
\text { décroissantes. }\end{array}$ \\
\hline 50 & 0,753 & 0,98 & 0,70 & Oscillations stabilisées. \\
\hline $6 e$ & 0,753 & 1,00 & 1,25 & Id. que $7 b$ \\
\hline $5 c$ & 0,750 & 1,40 & 0,75 & Oscillations stabilisées. \\
\hline $1 a$ & 0,749 & 1,48 & 0,45 & Id. que $7 \mathrm{~b}$. \\
\hline $\begin{array}{lll}5 & d\end{array}$ & 0,742 & 2,00 & 1,05 & Oscillations stabilisées. \\
\hline $6 d$ & 0,742 & 2,02 & 2,20 & $\begin{array}{l}\text { Au moment de la mesure les } \\
\text { oscillations etaient crois- } \\
\text { santes. Au cours des oscil- } \\
\text { lations, le distributeur at- } \\
\text { teint sa fin de conrse on- } \\
\text { verture et la puissance sur } \\
\text { l'arbre n'est pas constante. }\end{array}$ \\
\hline $7 a$ & 0,741 & 2,06 & 0,60 & $\begin{array}{l}\text { Id. que } 6 d \text {, mais sans at- } \\
\text { teindre la fin de conrse du } \\
\text { distributeur. }\end{array}$ \\
\hline $5 a$ & 0,740 & 2,18 & 1,70 & Id. que $6 d$. \\
\hline
\end{tabular}
les essais, d'un régulateur moderne très sensible. La cheminée est à section constante et ne comporte pas d'étranglement inférieur.

Le tableau ci-contre donne les valeur's de l'amplitude limite, mesurées sur place et calculées à l'aide de la formule (40).

La concordance est relativement bonne, les écarts étant presque toujours dans le sens de la sécurité. Parmi les raisons d'être de ces écarts, nous pouvons relever :

Variations de rendement avec l'ounerture du vannage. -- Dans la branche montante de la courbe de rendement, la stabilité se trouve accrue, et d'autant plus que la pente est forte. Les 
cing premiers essais se placent sur cette partic de la courbe. Pour les quatre restants, c'est exactement le contraire.

Surouverture. - Fin de course du vannage. -.. Le « pompage » étant fort, la fin de course est atteinte et la puissance n'est plus constante. Le phénomène s'écarte de son schéma théorique.

Pertes de charge dans les ouvrages d'amenée. - Mesurées vingt-cinq ans avant les essais, elles sont de l'ordre de $0,12 \mathrm{~m}$ pour le débit maximum turbiné. Une variation, même réduite, de ces pertes de charge, se traduit par une variation amplifice de l'amplitude donnéc par la formule $(40)$. Ainsi, pour $5 \%$ en plus ou en moins, l'amplitude varierait en sens contraire de $20 \%$ environ.
Il convient de citer enfin l'action du régulateur, ses recouvrements et jeux, dont notre formule ne tient pas compte.

Les formules (36 bis) et (37) donnent done une première approximation de l'ordre de grandeur de l'amplitude limite atteinte par les oscillations entretenues, mais elles n'éclairent nullement sur le temps requis pour arriver à ce stade.

Pour cela, il faudrait intégrer les équations pas à pas, ce qui est en général assez long à faire.

L'expérience nous a montré que, dans ce cas, il est préférable de faire appel au modèle réduit, qui, une fois mis au point, permet de faire de multiples vérifications et toutes sortes de manœuvres.

\section{VI. - LA SIMILITUDE DES CHEMINEES D'ÉQUILIBRE}

Cette similitude, dite de Durand [17] consiste à choisir les différentes échelles du modèle de facon que les rapports nature-modèle des paramètres :

$$
\frac{\mathrm{L}_{g} \mathrm{Q}}{f_{g} t} \quad \mathrm{Z} \quad \mathrm{P} \quad \mathrm{R} \quad \frac{\mathrm{Q}^{2}}{f_{0}^{2}}
$$

aient une même valeur. De même pour :

$$
\frac{\mathrm{F}_{c h} \mathrm{Z}}{t} \quad \mathrm{Q}
$$

Si les échelles sont désignées par :

$\lambda$ (longueurs galerie d'amenée),

Q (diamètres galerie d'amenée),

1) (diamètres cheminée d'équilibre),

ó (diamètre galerie au pied de la cheminée),

$\tau$ (temps),

$z$ (oscillations dans la cheminée),

$q$ (débit),

$\mathrm{K}^{\prime}$ (coefficient de perte de charge de la galerie),

$c$ (coefficient de rugosité de la galerie),

$\mathrm{K}^{\prime \prime}$ (coefficient de perte de charge du diaphragme),

$\Delta$ (diamètre du diaphragme),

de (40) et (41), on déduit :

i. $\varphi^{-2} q \tau^{-1}=\mathrm{K}^{\prime} q^{2}=\mathrm{K}^{\prime \prime} q^{2} \Delta^{-2}=z=q^{2} \delta^{-2}$

$$
\Phi^{2} z \tau^{-1}=q
$$

D'autre part :

$$
\mathrm{K}^{\prime}=\lambda c \varphi^{-4}
$$

En ce qui concerne $\mathrm{K}^{\prime \prime}$, il convient de re- marquer qu'on ne sait faire que des modèles ayant $K^{\prime \prime}=1$, c'est-à-dire qu'il faut adopter une même et seule échelle pour toutes les parties du raceordement galerie-cheminée pouvant influencer la valeur de la résistance de l'étranglement. En définitive, on a done :

$$
\begin{gathered}
\Delta=\delta=\Phi \\
\lambda \varphi^{-2} q \tau^{-1}=\lambda c q^{2} \varphi^{-4}=z=q^{2} \delta^{-2} \\
\Phi^{2} z \tau^{-1}=q
\end{gathered}
$$

soit quatre équations pour sept inconnues. On peut done choisir arbitrairement trois échelles.

Si on veut conserver sur le modèle le rapport des inerties de la cheminée et de la galerie, on a l'équation :

$$
z \Phi^{-2}=\lambda \varphi^{-2}
$$

ce qui réduit à deux le nombre d'échelles arbitrairement choisies.

Notons également que $\Phi$ peut ne pas être égal à $\delta=\Delta$, car en général le diamètre de la cheminée, pourvu qu'il soit suffisamment grand, n'a pas d’influence sur la résistance d'étranglement. Dans ce cas, le nombre d'échelles arbitrairement choisies est de quatre.

Ainsi, si nous considérons la figure 9, il suffit pour avoir $K^{\prime \prime}=1$, que toute la partie entourée par le trait soit représentée sans distorsion; si $\delta \neq \varphi$, on doit mettre un convergent ou un divergent à l'amont de la cheminée pour passer graduellement d'un diamètre à l'autre.

Ainsi, si l'inertie du tronçon à l'aval de la cheminée d'équilibre est important, il doit être également représenté sur le modèle. Les nouvelles 
quelles $\varphi_{c}, \lambda_{v}$ et $c_{t}$ se déterminent à partir des inuations :

$$
\lambda_{e} q \varphi_{c}^{-2} \tau^{-1}=z=c_{c} \lambda_{c} q^{2} \varphi_{c}^{-4}
$$

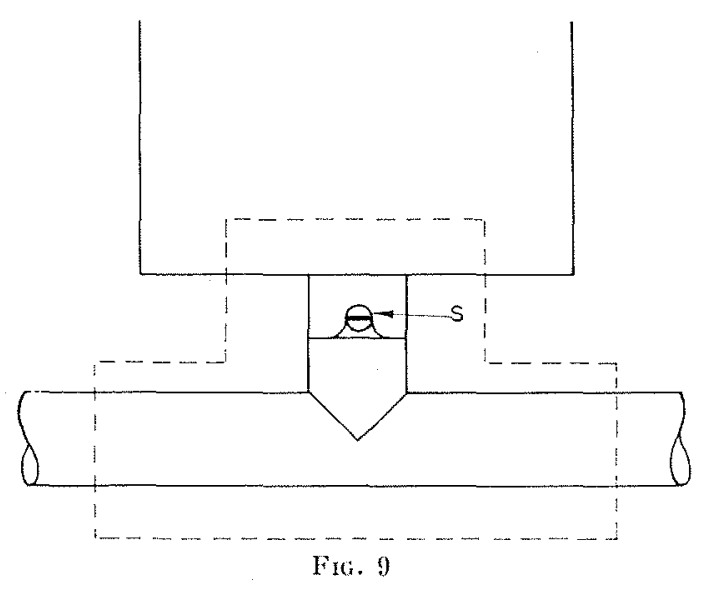

Finalement, pour reproduire les conditions aux limites, e'est-à-dire la loi de la forme (4) du débit turbiné, on se sert, au Laboratoire Dauphinois d'Hydraulique, d'un système breveté à panneau mobile sur lequel nous ne reviendrons pas car il a fait l'objet d'un article dans cette même revue [18]. La photographie figure 10 montre le panneau mobile du modèle de la cheminćc d'équilibre de Delcommune (Congo Belge), étudiée dernièrement par les soins du Laboratoire dans le but de déterminer les dimensions minima compatibles avec un amortissement rapide des oscillations.

Lorsque le système d'amenée comporte plusieurs cheminées d'équilibre, les épures d'intégration graphique deviennent très laborieuses, et les problèmes de stabilité compliqués. Dans ce cas, le modèle devient un instrument presque indispensable pour le bon dimensionnement des ouvrages.

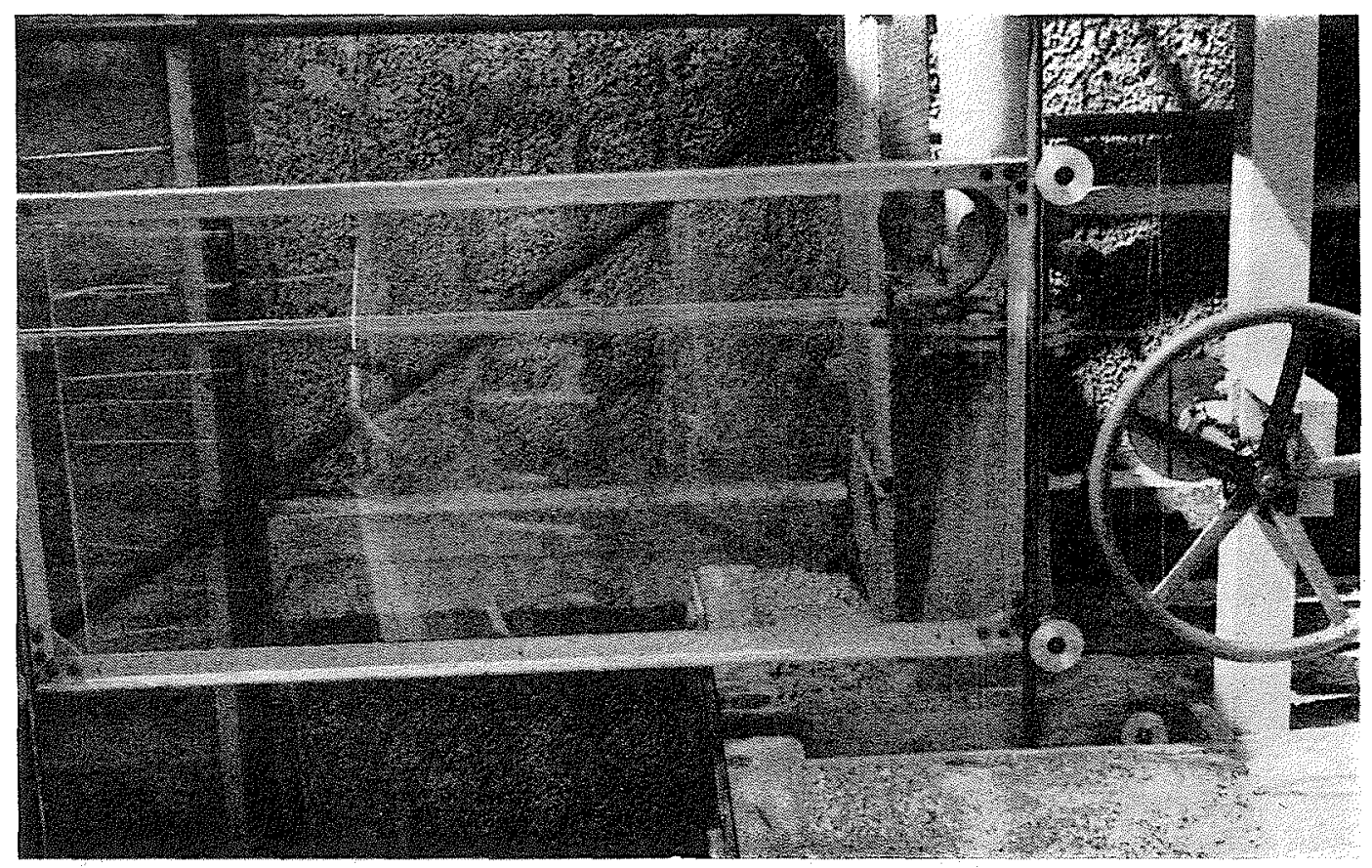

F1G. 10

\section{VII. - L'ETUDE DES DIAPHRAGMES DES CHAMBRES D'EQUILIBRE A ETRANGLEMENT}

Il est rare de voir de nos jours un projet de chambre d'équilibre ne comportant pas d'orifice diaphragimé à sa base, car on sait que l'économie sur le volume de la cheminée peut atteindre facilement 30 à $40 \%$.

Les ouvertures et les fermetures ayant des caractéristiques indépendantes, l'étranglement idéal est en général dissymétrique, la contraction étant plus faible dans le sens de la montée. Ceci vient en grande partie du fait que, bien qu'on envisage presque toujours la coupure totale du débit maximum, on exige rarement la mème chose à l'ouverture, du moins si la centrale comporte plus d'un groupe. D'habitude on 
se contente de charger un groupe à la fois, l'ouverture la plus défavorable étant la mise en route du dernier.

D’après sa définition mème, on voit qu'il est difficile, pour ne pas dire impossible, de calculer R. L'écoulement comporte en effet des changements de direction, contractions, expansions, etc., et seule une étude globale sur modèle réduit pernet de trouver les formes hydrauliques satisfaisant aux besoins du calcul. Ceci n'est que plus rai lorsqu'il s'agit de respecter des conditions différentes dans les deux sens de l'écoulement.

Nous allons évaluer d'une façon approchée la dissymetrie maximum qu'on peut demander à un diaphragme ne comportant pas des pièces mobiles, c'est-à-dire assurant une sécurité de marche optimum.

Admettons que nous décomposons $R$ en deux parties (fig. 9):

-.- La perte de charge par expansion à partir de la section contractée $\mathrm{cS}$, assimilable, en première approximation, à l'énergie cinétique : $\frac{\mathrm{Q}^{2}}{2 g c^{2} \mathrm{~S}^{2}}$.

- Le complément à $\mathrm{R}: \quad \alpha=\mathrm{R}-\frac{\mathrm{Q}^{2}}{2 g c^{2} \mathrm{~S}^{2}}$.
Si, de prime abord, nous négligeons \& par rapport à $R$, la dissymétric vaut:

$$
n=\left(\frac{c_{f}}{c_{0}}\right)^{2}
$$

Les valeurs thériques extrêmes étant:

$$
c_{j}=1 \quad c_{0}=0,5
$$

on tire $n_{\max }=4$, valeur qui ne constitue qu'une limite supéricure impossible à atteindre avec les formes d'usage courant.

On sera plus près de la réalité en prenant:

$$
c_{j}=0,95 \quad c_{0}=0,55
$$

d'où : $n \# 3$.

Si $\propto$ n'est pas négligeable, la dissymétrie vaut :

$$
n=\frac{\frac{\mathrm{Q}^{2}}{2 g c_{0}{ }^{2} \mathrm{~S}^{2}}+\alpha_{0}}{\frac{\mathrm{Q}^{2}}{2 g c_{f}{ }^{2} \mathrm{~S}^{2}}+\alpha_{f}}
$$

Si $\approx$ ne varie pas beaucoup avec le sens de l'écoulement et qu'on adopte :

$$
c_{f}=0,95 \quad c_{0}=0,55 \quad \alpha=0,2 \frac{\mathrm{Q}^{2}}{2 g c_{f} \mathrm{~S}^{2}}
$$

on trouve : $n=2,67$.

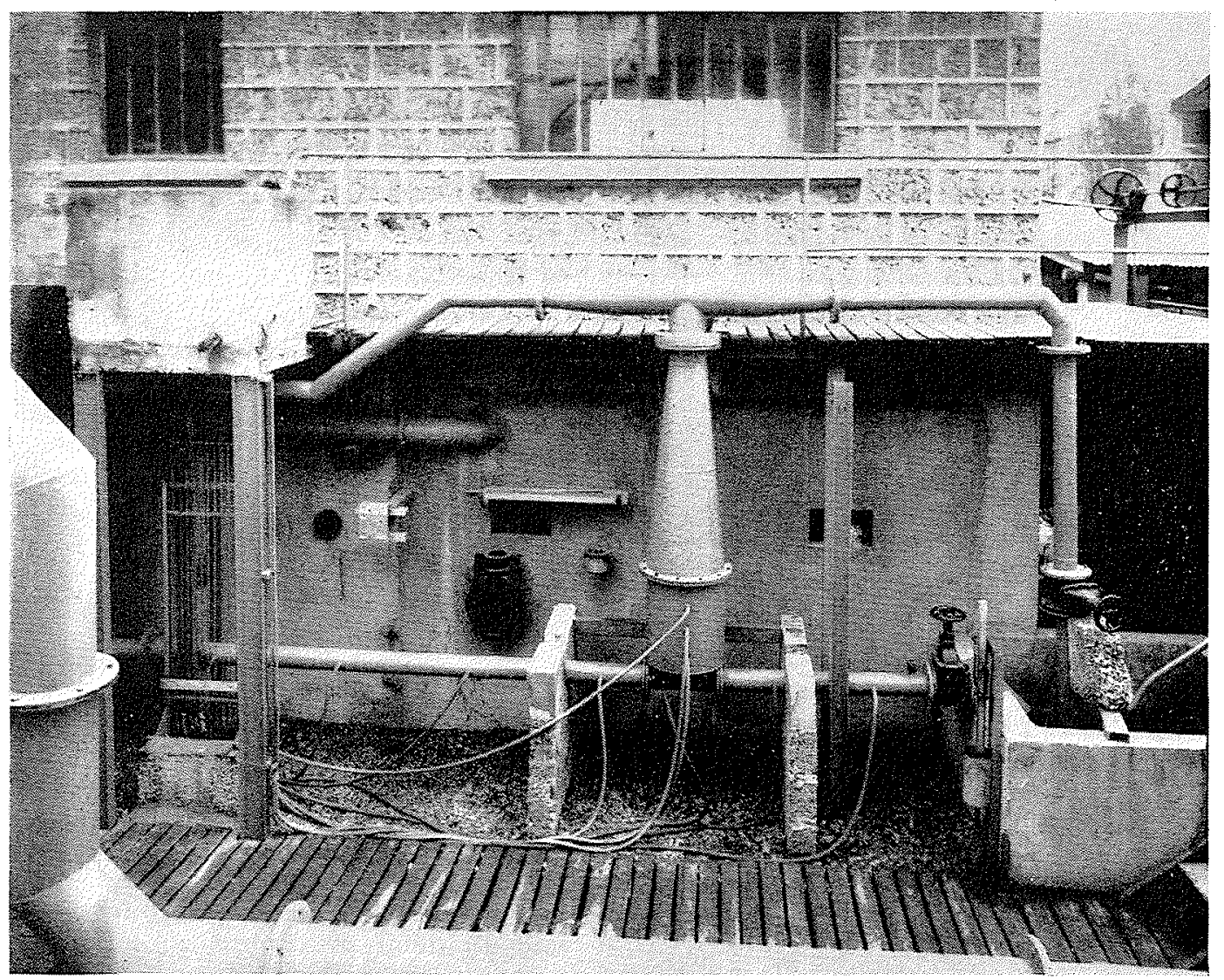

Fra. 11 
Cit rakul n'a aucune prétention à Texactitude, *if cependant il montre qu'il paraît difficile f vilinir une dissymétrie supérieure à 3 , sans 1: In recours à des formes tout particulièrement hi: il uludiées.

I. Laboratoire Dauphinois d'Hydraulique a (111) en service depuis longtemps un modèle 11. 11) pour l'étude des étranglements des 1. immbres d'équilibre. Ce modèle représente la Timmine et des tronçons de galerie à son amont Al if son aval, qui assurent sa bonne alimentafim. Les mesures se font en régime permanent, If ripartition et le sens des débits pouvant être imorlifés à volonté pour représenter tous les schémis possibles d'écoulement.

Parmi les cheminées d'équilibre étudiées dermirement, nous pouvons citer celles de :

Vauricio (Brésil); Afourer (Maroc); Rio Tertreto II (Argentine); Oued Agrioum (Algérie), etc.

Ces essais ont mis en relief les différences parlois notables qu'on trouve entre les valeurs données par le modèle et celles qu'on peut calculer par somme des diverses pertes de charge singulieves, en prenant des coefficients plus ou moins précis. D’ailleurs, même si chaque perte de charge pouvait être évaluée avec exactitude, leur somme ne donnerait pas la perte de charge tolale, car chaque singularité étant dans le sillage de la précédente, l'écoulement réel ne correspondrait pas au schéma de calcul.

Ainsi, à titre indicatif, nous pouvons remarquer que pour la cheminée d'équilibre d'Afourer on a trouvé qu'un diaphragme, dont le calcul avait donné $\mathrm{R}=14,30 \mathrm{~m}$, avait en réalité $\mathrm{R}=20,60 \mathrm{~m}$, soit une erreur de $44 \%$.

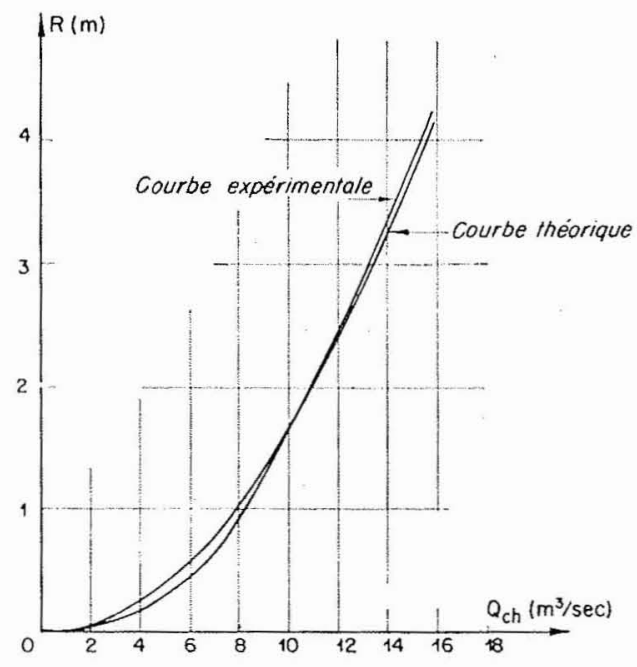

FIG. 12

Dans tout ce qui précède, on a admis que $R$ était donné par une loi $\mathrm{R}=k^{2} \mathrm{Q}^{2}{ }_{c h}, k_{2}$ étant une constante. En réalité, $k_{2}$ est fonction aussi de la répartition relative des débits, comme les essais l'ont mis en évidence; ainsi, si la cheminée est traversée par un débit donné, $R$ varie lorsqu'on

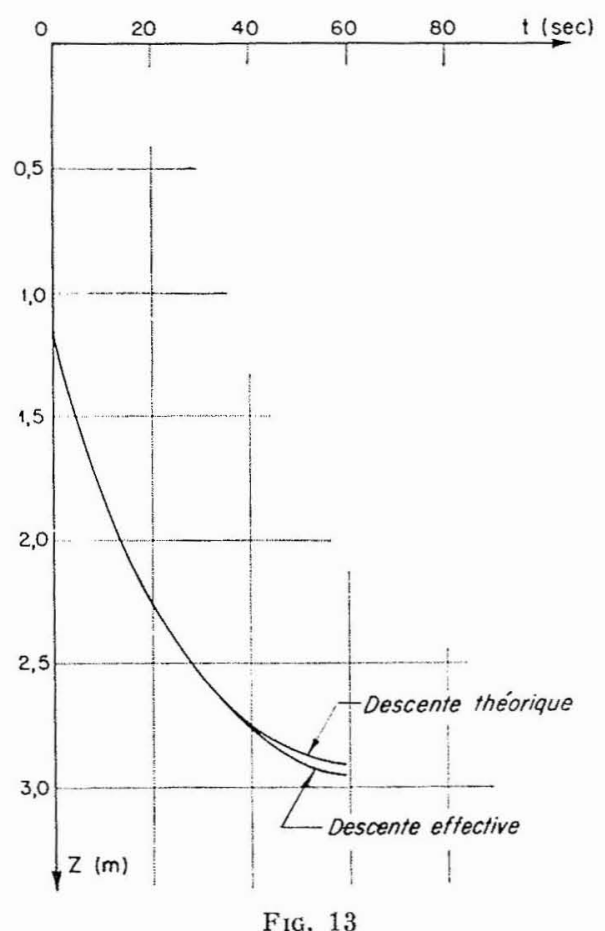

fait varier le débit dans la galerie d'amenée; il peut augmenter ou diminuer selon les caractéristiques de l'installation.

Si l'on veut bien faire les choses, avec la loi réelle de $R$, il faudrait faire une épure d'intégration graphique et vérifier l'amplitude de la première oscillation.

Ceci a été fait pour la chambre d'écuilibre de Rio Tercero II. La figure 12 donne deux courbes, l'une correspond à la loi parabolique théorique de $\mathrm{R}$ en fonction de $\mathrm{Q}_{c h}$, l'autre la loi réelle pour un débit turbiné égal au maximum. La figure 13 donne, pour les deux cas, la descente consécutive à une ouverture de 70 à $100 \%$ du débit turbiné $\left(^{\star}\right)$.

(*) Après avoir donné ce travail à l'impression nous avons pris connaissance d'un article de M. VuJica JevDEvic, « $O$ odredivanju gubitaka u prigusivacu kod vodostana 》, paru dans le $\mathrm{n}^{\circ} 2,1952$, de la revue yougoslave Elektropriveda.

Les essais faits pour la cheminée d'équilibre de Jajce II confirment ce que nous avons dit au sujet de l'influence de la répartition des détails sur la résistance d'étranglement. La courbe qu'il donne a une allure semblable à celle de notre figure 12 .

M. Jevdevic croit aussi, comme nous, que le calcul est impuissant à lui tout seul pour dimensionner correctement un diaphragme placé au pied d'une cheminée d'équilibre. 


\section{VIII. - DIFFERENTS PROBLEMES QUI FONT APPEL AU MODELE REDUIT}

Nous passerons en revue quelques problemes concernant les cheminées d'équilibre, qui ont été résolus à la suite d'une étude sur modèle faite au Laboratoire Dauphinois d'Hydraulique.

Cheminée d'Égullibre de Monistrol-d’Allier.

Ouvrage déversant où l'on voulait éviter un déversement libre car on craignait que le vent ne déplace la lame d'eau pour la faire tomber au-delà du canal d'évacuation.

La figure 14 montre les détails d'un modèle partiel reproduisant seulement un secteur de $30^{\circ}$ qui permit d'établir que la mise en place d'un chapeau déflecteur collait la lame déversante contre les parois de la cheminée et évitait l'action nuisible du vent.

Un modèle d'ensemble (fig. 15), ainsi que l'ouvrage lui-même (fiğ. 16) confirmèrent les résultats des essais par le modèle partiel.

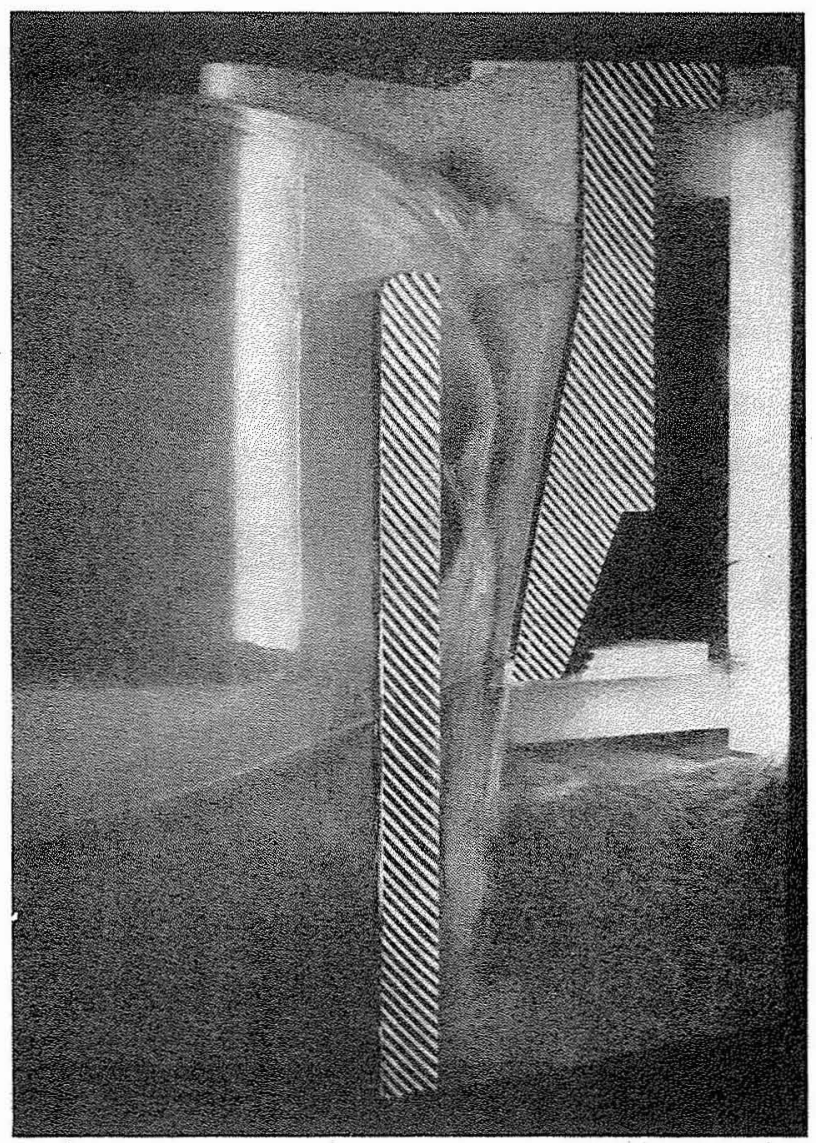

FIG. 14

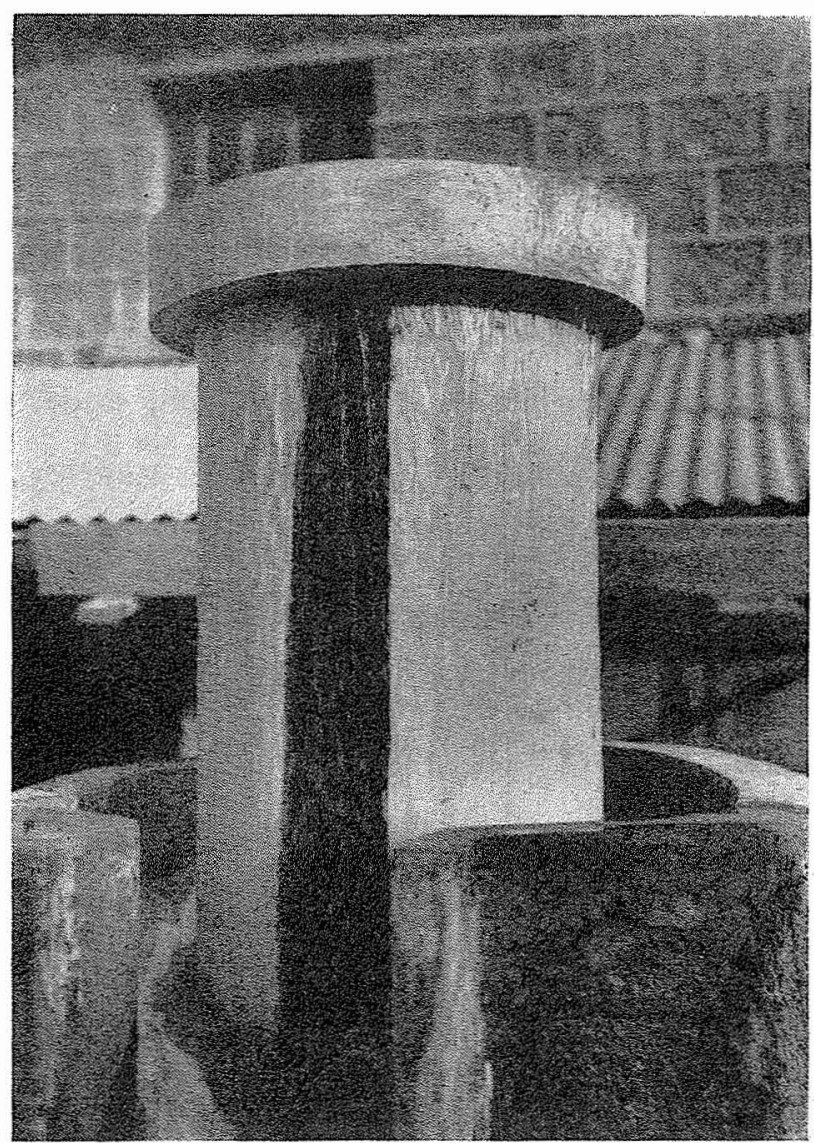

Fic. 15

Cheminée d'éguilibre du péage de Vizille.

Il s'agissait de réduire au minimum les dimensions du déversoir de cette cheminée. Les essais permirent de mettre au point un déversoir en « bec de canard » (fig. 17) qui donnait à l'essai pleine satisfaction.

\section{Cheminée D'Éouillibre D'Afourer}

(premier projet).

Il s'agit d'une chambre d'équilibre à étranglement comportant deux expansions, l'une en haut, l'autre en bas. Le diaphragme ayant été convenablement dimensionné, deux difficultés restaient à surmonter :

- Lors de l'ouverture, à la fin de la vidange de la chambre d'expansion inférieure, il risquait de se produire un vortex suscepti- 


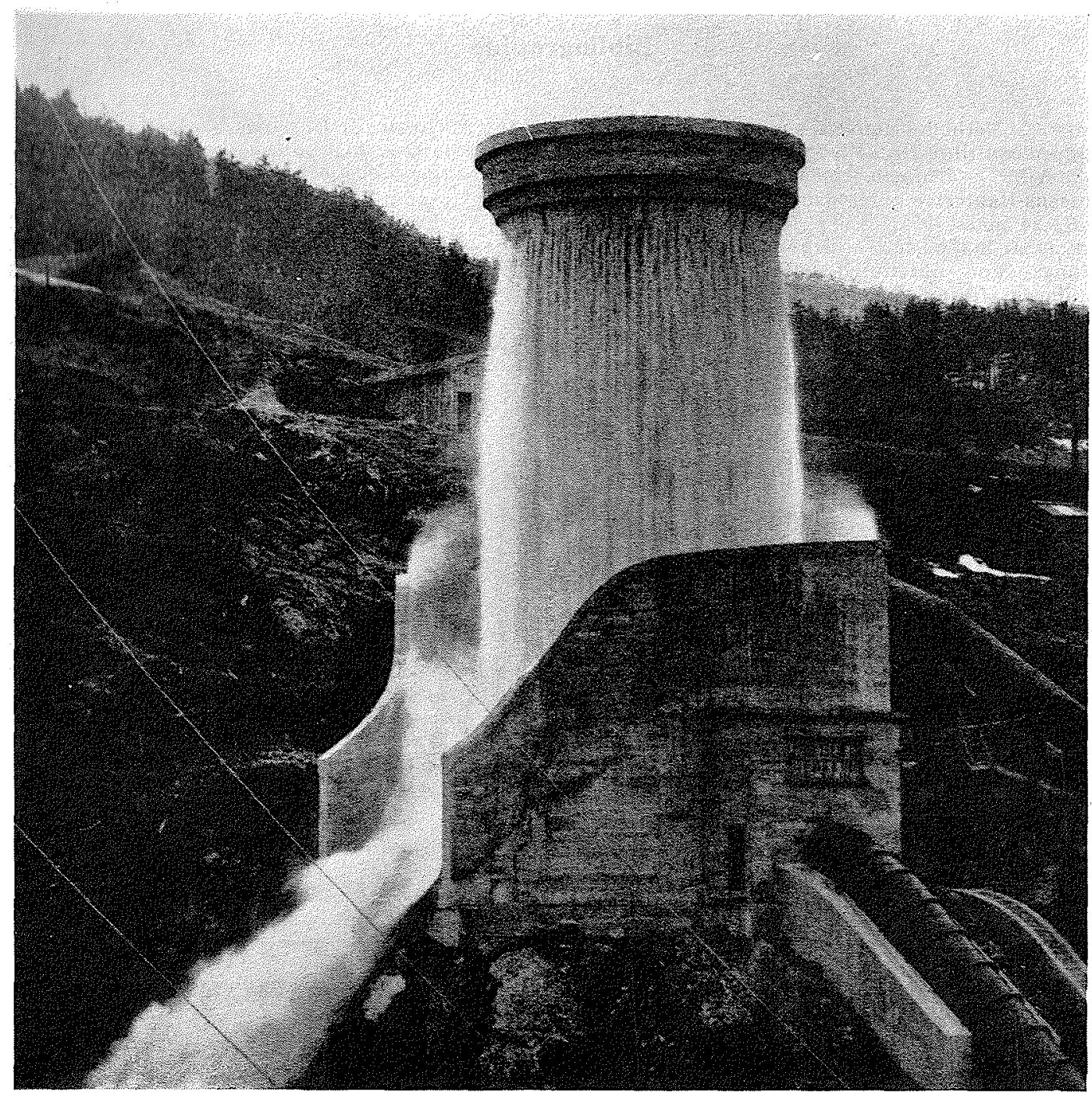

FiG. 16

ble d'entrainer de l'air jusqu'aux conduites forcées;

-.. Lors de la fermeture, le jet issu du diaphragme, insuffisamment amorti par le matelas d'eau rencontré, risquait de créer une grosse intumescence-champignon audessus de la surface libre, susceptible, en s'élevant, d'emprisonner provisoirement de l'air dans l'épanouissement inférieur.

\section{IX. - LES CONDITIONS D'EXPLOITATION ET LEUR INFLUENCE SUR LE CALCUL DES CHAMBRES D'EQUILIBRE}

Il est d'usage de dimensionner les cheminées d'équilibre pour satisfaire à une coupure totale et une ouverture partielle de $n$ à $100 \%$, toutes deux instantanées.'On vérifie ensuite les oscillations consécutives aux manouvres qui pourraient vraisemblablement se produire en cours d'exploitation.

Il est d'une grande importance que l'auteur du projet soit aussi bien renseigné que possible sur les caractéristiques du réseau, car si un man- que de prévision peut produire des dégâts importants, une hypothèse de calcul par trop pessimiste conduirait à un surdimensionnement inutile et souvént coûteux.

Chaque cas étant différent des autres, on ne saurait donner des lois fixes, l'intuition et l'expérience de l'Ingénieur devant lui permettre de peser chaque fois les facteurs en jeu et de tenir compte de tous pour arriver à un dimensionnement optimum de la cheminée d'équilibre. 


\section{Bibliographie}

[1] L. Escande. - Méthodes nouvelles pour le calcul des chambre d'équilibre. Dunod, Paris, 1950.

[2] J.F. Bnutrix. - L'aménagement hydro-électrique de Rossens-Hauterive après deux ans d'expérience. B.T.S.R., 24 mar's 1951 .

[3] J. Frank et J. Schüller. - Schwingungen in den Zuleitungs- und Ableitungkanälen yon Wasserkraftanlagen. Julius Springer, Berlin, 1938.

[4] J. Calane et D. Gades. - Théorie des chambres d'écquilibre. La Concorde, Paris et Lausanne, 1926.

[5] J. Carane et D. Gaden. - Calcul d'une chambre d'équilibre à grands épanouissements supérieur et inférieur à l'aide des « valeurs relatives». Revue Générale de l'Electricité, n" 26, 1926.

(6) A. Schormitsch. - Graphische Hydraulik. Sammlung mathematisch-physikalischer Lehrbücher. Vol. 21. B. G. Tenbner, Leipzig, 1923.

[7] T. Schirman. - Zeichnerische Verfahren zu: Darstellung der Schwingungen in Kammer- und Uberfallwasserschlossern. Wasserkraft und Wasserwirtschaft, $\mathrm{n}^{\circ} 3,1942$.

8] E. Des Felice. - Accorgimenti pratici per la determinazione grafica delle oscillazioni nelle camere di equilibrio di qualsiasi tipo. L'Energia Elettrica, $\mathrm{n}^{\circ} 4-5,1947$.

[9] M. Bouvard et J. Monbert. - Méthode graphique pour le calcul des cheminées d'équilibre. La Honille Blanche, $n^{\circ} 5,1950$ et 3,1951 .
[10] J. Calane et D. Gaden. - De la stabilité des installations hydrauliques munies de chambres d'équilibre. Schweizerische Balzeitung, 30 juillet et 6 août 1927.

11] G. Evangelist1. - Pozzi piezometrici e stabilità di regolazione. L'Energia Elettrica, $\mathrm{n}^{\circ}$. 5 et 6, 1950.

12] E. Scmeni. -- Sulla validità della regola di Thoma per le vasche di oscillazione degli impianti idroclettrici. L'Energia Elettrica, no 11-12, 1947.

[13] A. GHETrt. - Ricerche sperimentale sulla stabilita di regolazione dei gruppi idroelettrici con derivazione in pressione e pozzo piezometrico. L'Energia Elettrica, n० 11,1951 .

14] Discussion on "Hydraulic Stability", by A.W.F. McCues. Engineering Journal of Canada, octobre 1933.

[15] Ch. JaEger. - De la stabilité des chambres d'équilibre et des systèmes de chambres d'équilibre. Schweizerische: Bauzeitung, 20 novembre 1943.

[16] L. Escande. - Etude de la stabilité des chambres d'équilibre à étranglement. Le Génie Civil, $\mathrm{n}^{\circ \mathrm{s}} 12$ et $13,1951$.

[17] W.F. Deravid - Application of the law of kinematic similitude to the surge-chamber problem A.S.M.E. Paper, n ${ }^{\circ} 1827,1921$.

[18] Mlle CuÉxinst. - Stabilité des cheminées d'équilibre. Rappel très sommaire des éléments de ce probleme. La Honille Blanche, $\mathrm{n}^{\circ}$ 6, 1948.

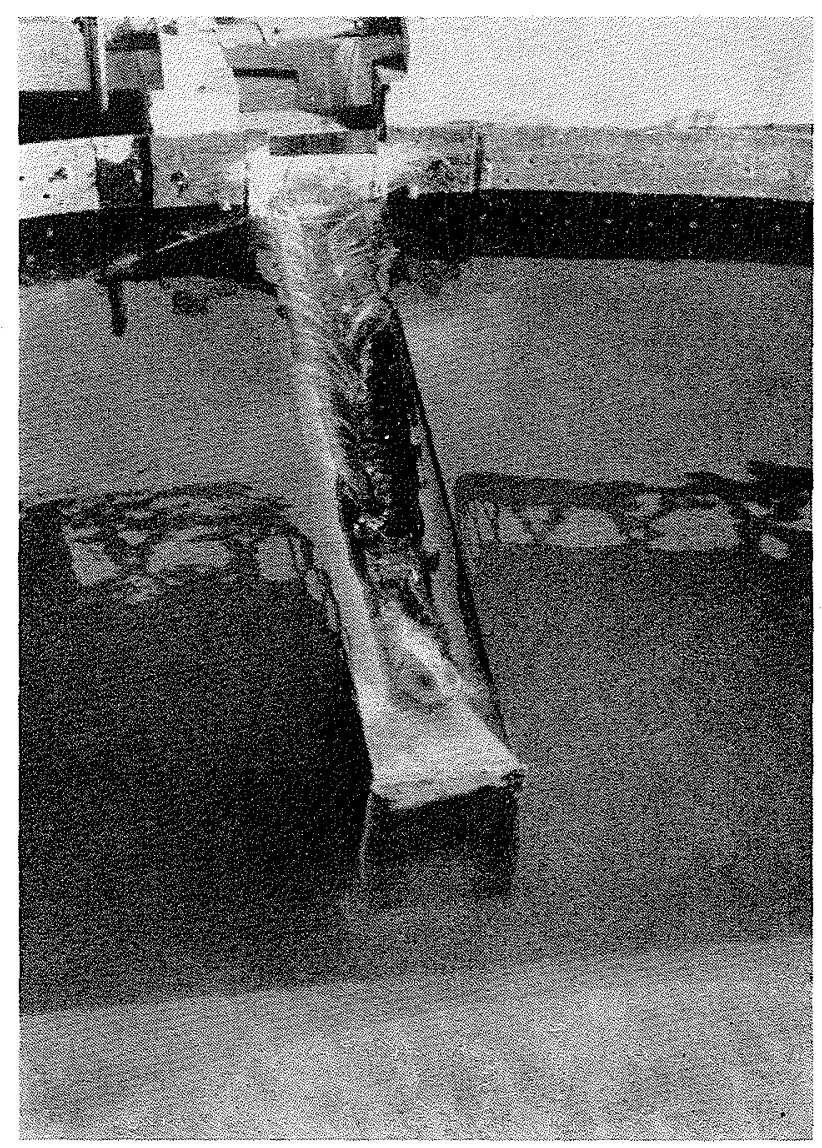

FIG. 17 